\title{
Sensitive periods in human social development: New insights from research on oxytocin, synchrony, and high-risk parenting
}

\author{
RUTH FELDMAN \\ Bar-Ilan University
}

\begin{abstract}
Sensitive periods (SP) in behavioral development appeared in the biological sciences during the first decade of the 20th century, and research in animal models beginning in the 1950s provide terminology and evidence for SP effects. This paper proposes a rigorous program for human SP research and argues that the complexity of the human brain and variability of the human ecology necessitate that SP effects must be studied in humans, employ longitudinal designs starting at birth, test mechanism-based hypotheses based on animal studies that manipulate early environments, and utilize high-risk conditions as "natural experiments." In light of research on the molecular basis of critical periods and their sequential cascades, it is proposed that the oxytocin (OT) system, an ancient and integrative system that cross-talks with the stress, reward, immune, and brain stem mediated homeostatic systems and supports mammalian sociality, plays a unique role in experience-dependent plasticity that buttresses SP effects due to its (a) dendritic mode of release leading to autoregulated functioning primed by early experience, (b) pulsatile pattern of activity, and (c) special role in neural plasticity at the molecular and network assembly levels. Synchrony, the coordination of biology and behavior during social contact, is suggested as a mechanism by which SP exert their effect on OT functionality, the social brain, and adult sociality. Findings from four high-risk birth cohorts, each followed repeatedly from birth to 10 years, provide unique "natural experiments" for human SP research based on specific programs in animal models. These include prematurity (maternal proximity), multiple birth (peer rearing), postpartum depression (low licking and grooming), and chronic unpredictable trauma (maternal rotation, variable foraging demands). In each cohort, hypotheses are based on the missing environmental component during SP, and findings on social synchrony, OT functionality, stress response, emotion regulation, and mental health accord with the multilevel and dynamic principles of developmental psychopathology. The results on the potential for reparation versus chronicity following early deprivation highlight a flexible conceptualization of resilience based on human SP research. Consideration of SP effects at the molecular, endocrine, brain, and behavioral levels and in relation to the neural plasticity and multifinality of human social functions may assist in fine-tuning early detection and the construction of targeted individualized interventions.
\end{abstract}

In the punctual center of all circles white Stands truly. The circles nearest to it share

Its color, but less as they recede, impinged By difference and then by definition

As a tone defines itself and separates

And the circles quicken and crystal colors come And flare and bloom with his vast accumulation Stands and regards and repeats the primitive lines.

From the Packet of Anacharsis, Wallace Stevens

As the biological sciences became the central lens through which humans comprehended their world, assuming the position previously occupied by philosophy, Western thought shifted from understanding the universe in terms of hierarchical order or incremental progress, for which a "chain" or "line" may be the best underlying metaphor (see Lovejoy, 1936), to viewing the universe in terms of systems, for which the circle is the most fitting symbol. Reflected in Stevens's

Address correspondence and reprint requests to: Ruth Feldman, Department of Psychology and the Gonda Brain Sciences Center, Bar-Ilan University, Ramat-Gan, Israel 52900; E-mail: Feldman@mail.biu.ac.il. poem (quoted in Bloom, 1980) is the notion that systems, or circles, have several important features: their center contains their most important qualities in a concentrated form; as rings grow farther away from the center, their "color" or qualities become integrated with foreign elements, mainly those outside the system; repetitions of "programs" encoded within the core reverberate throughout the circle rings; and all components of the system (all dots on the circle) are interrelated and constrained by the same external limits. Systems develop and reorganize, and colors become more pronounced and system specific from the initial "white." However, unlike the line, circles are not infinite but self-contained, grow from the core outward, follow a finite set of rules, and execute a predetermined series of programs, which, given adequate tools, can be observed already in their most embryonic form. Systems, therefore, contain sensitive periods (SP) by definition. Embedded in modern science is the assumption that getting to the root of systems and unraveling the laws of their SP can bring us closer to understanding the universe.

Of all living systems, humans are by far the most complex, their development most extended, and the experience-dependent pathways to human cognitive functions involve the greatest plasticity. Research on SP in humans must therefore 
draw on observations made in other organisms, including animal models and the simplest living organisms (Knudsen, 2004; Rice \& Barone, 2000; Takesian \& Hensch, 2013). Yet SP in humans are more elusive and brain functions are the end product of multiple pathways, limiting our capacity to describe time windows with the required precision for a full-blown critical period effect and charting developmental psychopathology as a scientific field where multifinality and equifinality are key constructs (Cicchetti \& Toth, 2009) and nonlinear progress the typical process (Cicchetti \& Tucker, 1994). Moreover, clear causal links between a defined window in the development of a brain structure, specific environmental inputs required to sustained it, and resulting lack of function in its failure, requirements for the classic "critical period" effect, are rare at best in humans. Due to the great plasticity of the human brain, there are also more opportunities for reorganization and repair following initial deprivation, highlighting the need for a sensitive-period perspective on resilience. From a developmental psychopathology perspective, it is important to define the qualities that enable some children to benefit from opportunities after initial deprivation, form measurements that predict individual differences in self-organizing trajectories, and define components that must still exist in the early environment to make reparation possible (Cicchetti, 2013; Cicchetti \& Tucker, 1994; Feldman \& Eidelman, 2009a; Roth \& Sweatt, 2012).

One extraordinary example of reparation following extreme early deprivation is depicted in the autobiographical posthumous book The First Man (1994) by Nobel Prize winning author Albert Camus. Camus, an existential philosopher and author, was raised without a father by a deaf-mute illiterate mother in war-torn Algeria under conditions that can only be described as extreme childhood adversity, including physical abuse, intellectual and material poverty, aggressive discipline, a dangerous neighborhood, and political strife. Camus describes in exquisite tenderness both the nonverbal, "touchbase" affectionate bond with his mother and a meaningful attachment relationship at the transition to adolescence with a teacher-mentor who introduced him to the world of letters and opened opportunities for later education. As such, a central question for the study of neural plasticity, SP, and resilience in the 21 st century is whether there are essential biobehavioral experiences that trigger specific neurological or endocrine systems impacting on gene expression, brain development, and social fittedness that must still exist in the early environment for reparation to be possible. The central hypothesis proposed here is that while inborn biological and genetic factors certainly differentiate the genius from others, the nonverbal early synchrony Camus experienced with his mother provided essential inputs during a SP for social growth without which later provisions could not have fully repaired even in the most plastic brain.

Consistent with these notions, the goal of the current paper is to (a) chart a program for rigorous research on SP in humans by offering a human-specific conceptualization, (b) describe the critical role of the oxytocin (OT) system in sup- porting neural plasticity at multiple levels from single neuron to intergroup, (c) highlight synchrony as a central mechanism in this process, and (d) utilize four high-risk birth cohorts, each followed across the first decade of life, as distinct windows to formulate mechanism-based hypotheses on SP in human social development. Because most research on the role of OT in neural plasticity, affiliative bonding, social functions, and psychopathology emerged in the last decade, the OT system can provide a timely and innovative viewpoint on SP and neural plasticity in humans not available to theories on plasticity 20 years ago.

\section{Program for Rigorous Research on SP in Human Social Development}

A rigorous research program on SP in humans must move away from the global assumption that early adversity leads to unfavorable outcome to testing mechanism-based hypotheses on the basis of human-specific conceptualization and relevant findings in animal models. Current research on the molecular basis of critical periods, particularly the classic paradigm of ocular dominance, provides a timely perspective that can be integrated into human research (Toyoizumi et al., 2013). According to Hensch (2005), critical periods (CP) are defined as strict time windows during which experience provides information that is essential for normal development and permanently alters performance. In comparison, SP are limited times in development during which the effect of experience on brain function is particularly strong. The "softer" notion of SP does not imply that a function is completely eliminated but suggests that the brain may take atypical "detours" when essential experiences are unavailable or not consistently offered. Such detours may appear under stressful life events, unpredictable circumstances, or difficult task requirements. This suggests that teasing apart early risk conditions by charting the specific environmental elements missing in each condition may provide a useful window to study SP in humans. Because a CP involves a sequence of sensitivities to increasingly complex aspects of the environment, which correspond to predetermined developmental sequences from lower to higher brain structures, assessing the effects of early experience on later functioning should begin at birth or as close to birth as possible and assess how children meet later $\mathrm{SP}$ in development. In addition, because much of adult behavior reflects functioning of neural circuits sculpted during early SP (Elbert, Pantev, Wienbruch, Rockstroh, \& Taub, 1995; Kim, Relkin, Lee, \& Hirsch, 1997) and the great variability of the human ecology makes comparisons between early experiences impossible, research on human SP should be conducted on the individual using prospective longitudinal designs.

Recent work on CP in sensory development at the molecular level indicates that the transition from a pre-CP to $\mathrm{CP}$ time window occurs through inhibitory mechanisms involving GABAergic processes that preferentially select relevant information from the spontaneous input available in the envi- 
ronment (Hensch et al., 1998; Toyoizumi et al., 2013). CP occurs via alterations in the degree of plasticity in neural circuits as they become increasingly sensitive to external stimuli, resulting in a cascade of $\mathrm{CP}$ from low to high functioning. A CP in sensory development opens when there is a switch from internally driven spontaneous activity to externally driven evoked activity.

Similar principles may apply to social neuroscience. The neural systems that underpin social affiliation evolve through the OT system's increasing sensitivity to the recurring social elements in the environment, enabling a growing familiarity with the attachment figure and imbuing specific elements in mother and environment with incentive value (Feldman, 2012a; Insel, 2010; Ross \& Young, 2009). Similar to the classic ocular dominance effect, when the visual cortex reorganizes toward input from the open eye following a period of transition, deprivation of essential elements in the social environment, or even significant reduction, as seen in infants of "low licking and grooming" dams (Weaver et al., 2004), reorganizes OT receptor availability in the neocortex and critical limbic sites (Zheng et al., 2014). OT availability at these sites plays a key role in mammalian sociality and enables infants to form preferences toward cues required for navigating their social niche and forming dyad-specific attachments (Carter, 2014; Kendrick, 2013). During the postbirth period, OT receptors become connected to specific social cues via the experience-dependent plasticity of the OT system and its links to the brain dopamine (DA) reward system (Gimpl \& Fahrenholz, 2001), the olfactory-amygdala pathways (Ferguson, Aldag, Insel, \& Young, 200), innervation of the sensory cortices (Zheng et al., 2014), and a sharpening signal to noise ratio in hippocampal pyramidal cells (Owen et al., 2013), thereby shaping the brain's social perception, preferences, and memory.

Critical for human SP research is that processes related to OT functionality are highly species specific (Goodson, 2013). The OT system is among the most evolutionary-ancient and conserved systems, implicated in thermoregulation, water economy, and day-night rhythmicity in invertebrates (Beets, Temmerman, Janssen, \& Schoofs, 2013), regulatory processes in worms (Donaldson \& Young, 2008), mate selection and flocking in birds (Adkins-Regan, 1998), exclusive bonding in herding animals (Keverne \& Kendrick, 1992), and social affiliation in rodents (Insel \& Young, 2001), primates (Maestripieri, Hoffman, Anderson, Carter, \& Higley, 2009), and humans (Feldman, 2012a). Evolutionary constraints guided this flexible and environment-dependent system to direct young to the salient cues in their surrounding that will help them bond with their parents, function within their ecology, and engage in the social structure of their species (Keverne \& Kendrick, 1992). Neurobiological and behavioral processes of social bonding are also species specific. While rodents rely solely on olfactory cues for activating the OT system (Ferguson et al., 2001; Sanchez-Andrade \& Kendrick, 2009), olfactory processes and their neuropathways are less critical for humans. The distribution of OT receptors in the brain is also species specific (Hammock, 2014), with substantial differences in receptor localization not only between rodents, primates, and humans, but also within a rodent species, for instance, between monogamous and polygamous subtypes (Ross \& Young, 2009). Thus, before adapting findings from animal models, the results must be tested in humans. It is also noteworthy that, unlike animals in nature who mainly carve a fitted ecology for their young, human-made ecologies often fail the needs of the human infant. Consider the number of infants growing up without the essential nutrients required for social-emotional growth, because of conditions such as prematurity (10\% of births globally), postpartum depression $(15 \%)$, and the millions of infants growing up in war zones, poverty pockets, unstable housing, or violent neighborhoods, and deprived of optimal rearing conditions that may render later reparation impossible or partial.

Despite the species specificity of OT and social processes, animal research on the molecular and cellular basis of $\mathrm{CP}$, the maturational processes leading to and terminating $\mathrm{CP}$, and the links between defined brain structures and external inputs is essential for two main reasons: it generates specific hypotheses whose parallels can be tested in humans, and it coins concepts that can provide the language for SP human research. The following six parameters are important in order to advance rigorous research on human SP, particularly as it pertains to social-emotional growth, mental health, and the experience-dependent plasticity of neurobiological systems that support social functioning.

1. Human-specific research: Length and extent of $\mathrm{CP}$ in mammals depend on growth trajectories and life expectancy of the species (Toyoizumi et al., 2013). The complexity of the human brain, the large neocortex, the multiple pathways to brain growth, and the great variability of the human ecology impacting on the social brain (Keverne, 2013), render human research a must before conclusions from animal models can be applied. Mechanismbased hypotheses, while drawing on findings from other species, must be tested in humans.

2. Mechanism-specific approach: Studies of human SP must test very specific mechanistic hypotheses based on animal research that link the missing ingredient in the infant's environment with the specific disruptions to later functioning via the effects of these missing (or excessive) elements on well-defined neurobiological systems that underpin the outcome (Feldman, Rosenthal, \& Eidelman, 2014). Such research must differentiate one adverse condition from another and, by charting mechanisms that underpin each condition, examine differential effects not only on global outcomes but also on specific neurobiological, regulatory, and social processes.

3. Prospective longitudinal studies: To test mechanismbased assumptions linking altered early environments and later dysfunction, research must utilize longitudinal designs. Due to the multifinality and equifinality of human social development (Cicchetti \& Toth, 2009), SP hypoth- 
eses should be examined on the individual. Moreover, because most SP involve the perinatal and early infancy periods, longitudinal research should begin at birth, or as close to it as possible, involve multiple testing, and, because of the long maturation of our species, follow children over lengthy epochs. Unfortunately, studies linking adult pathology to early adversity often rely on retrospective accounts that are colored by current state and the imperfection of human memory.

4. Role of specific neurobiological systems: Testing the way specific neurobiological systems are shaped by the environment during SP and over time sculpt brain and behavior is required for understanding how SP exert their effect. Different neurobiological systems support distinct maturational processes, play a role in different stages of pre- and postnatal development, and require unique environmental inputs for smooth functioning. Different neurobiological systems also have specific roles in brain plasticity, singly or via crosstalk with other systems (Carter, 2014; Donaldson \& Young, 2008; Roth \& Sweatt, 2012; Weaver et al., 2004). There is thus benefit for addressing human SP from the perspective of a single neurobiological system or the interchange between several systems in supporting functioning or hampering growth.

5. Careful observations of the highly variable human environment: Because of the immense variability of the human ecology, it is impossible to detail the nature of early provisions in a manner similar to animal models. Deprivation is typically not complete, stressors are not uniform across subjects, and provisions vary by nature, intensity, and timing (imagine, for instance, the tremendous variability of the term "maternal sensitivity"). It is thus essential to find novel ways to characterize "environmental inputs," including direct observations in the natural ecology, microcoding of behavioral processes, and observations of the growing child across multiple daily tasks and contexts and in situations that demand diverse emotional and functional response.

6. Utilizing high-risk conditions as "natural experiments" for human SP research: Ethical constraints preclude experiments that manipulate the early environment, particularly with regard to consistent and adequate caregiving. Human research must, therefore, utilize naturally occurring conditions that involve substantial alterations and measurable early deprivations of parental care and chart the parameters of such changes. An important feature often missing from longitudinal research is an attempt to tease apart independent risk conditions from a set of comorbidities. For instance, premature infants, apart from immature brain growth and maternal deprivation during incubation, also have a greater chance to be born as part of a multiple gestation, to single mothers, suffer intrauterine substance abuse, and be reared by depressed mothers. Hypotheses on SP effects should use these "natural experiments" as cleanly as possible by trying to disentangle risk conditions and propose hypotheses on the basis of animal studies that match the specific condition on critical parameters. To date, much human research on early adversity is too global in characterizing deprivations, separating comorbidities, or testing mechanism-based predictions.

In the following sections I describe the findings from four longitudinal birth cohorts, each followed from birth and across the first decade of life. In each cohort, the missing environmental input is detailed on the basis of specific research programs in animal models, which provide the window to hypothesis testing and interpretation. The results related to the role of OT in shaping brain-behavior links in light of the specific missing element are presented. Across cohorts, repeated assessments of the rearing environment were conducted, utilizing microcoding of interactions with mother and father and observations of child emotion regulation and social skills in multiple tasks and contexts. Biobehavioral synchrony is highlighted as an overarching mechanism by which the early environment exerts its effect through the coordination of biological and social processes during social contact (Feldman, 2007a, 2007b, 2007c, 2013). Finally, in each condition, later developmental difficulties, arrests, or reparations are measured across domains in relation to the missing component during SP.

The OT system is highlighted as a model system for studying how early experiences sculpt the brain during SP for social growth. It is suggested that the OT system is particularly relevant for SP research for three main reasons, which are further discussed below: (a) its special mode of functioning in the brain as both neurotransmitter and hormone, and its unique features involving both central and dendritic release that enable long half-life and activity at locations far from OT-producing sites; (b) its pulsatile nature of activity, which enables experience-dependent rewiring of the social brain; and (c) its involvement in processes of brain plasticity at both the molecular and network assembly levels. Most important for its role as a critical neurobiological system mediating environmental effects on the developing brain is that OT is a highly integrative system. OT is not only closely linked with, and at times interchangeable in terms of receptor affinity, with the arginine vasopressin (AVP) system (Carter, 2014; Weisman, Schneiderman, Zagoory-Sharon, \& Feldman, 2013) but also maintains online crosstalk with the hypothalamuspituitary-adrenal (HPA) axis (Dabrowska et al., 2011; Smith \& Wang, 2012; Weisman, Zagoory-Sharon, \& Feldman, 2013), DA (Love et al., 2012; Scheele et al., 2013), and immune (Clodi et al., 2008; Yaniv et al., 2015) systems; its receptors are widely distributed throughout body and brain, including the heart, pancreas, gut, and sexual organs (Gimpl \& Fahrenholz, 2001); and it is an epigenetic system by nature that is particularly open to early environmental effects (Cameron et al., 2008; Szyf, McGowan, \& Meaney, 2008). Evidence suggests that connections among the OT and DA (Carter, 2014) and immune systems (Higashida, Yokoyama, Kikuchi, \& Munesue, 2012) tighten in the face of stress or in the context of maternal care (Shahrokh, Zhang, Diorio, 
Gratton, \& Meaney, 2010). It thus appears that OT plays a critical role in shaping SP effects by integrating body and brain, individual and environment, and in binding the physiology and behavior of conspecifics into a shared time-locked event, that is, biobehavioral synchrony (Feldman, 2012a). Moreover, OT is critically involved in plasticity at the cellular, molecular, and brain levels, and promotes neurogenesis in the adult brain even under condition of heightened stress (Leuner, Caponiti, $\&$ Gould, 2012). The close yet nonlinear relations between the affiliation and stress system, between OT and the HPA axis, both originating in hypothalamic nuclei, enable the oxytocinergic system to function as a buffer against the immediate effects of environmental stress on the developing brain as well as against the effects of early stress on later growth (Neumann, 2008). In light of these connections, in each cohort, I will also describe findings related to the HPA system and, when available, other stress-related hormones interacting with cortisol (dihydroepiandrosterone [DHEA] and salivary alpha amylase [sAA]). The four cohorts and their reference to specific animal program are presented in Figure 1.

As seen, the four birth cohorts describe high-risk conditions affecting a large number of infants globally: prematurity, multiple birth, maternal postpartum depression, and unpredictable trauma. Each cohort was seen across the first decade of life in multiple observations with mother and father, emotion-regulatory paradigms, hormonal assessments, and psychiatric evaluation. The missing component from the rearing environment in each condition is based on specific empirical programs in animal models: maternal proximity/ deprivation (prematurity), exclusive parenting/peer rearing (multiple birth), diminished maternal behavior/low licking and grooming (maternal postpartum depression), and inconsistent care/maternal rotation or variable foraging demand (repeated trauma).

\section{Early Animal Studies on CP in Social Development Pave the Way for Modern Neuroscience Research on SP in Humans}

To trace the origins of research on SP in social development, one must go back more than a century. While the "classical" CP research focused on sensory and motor development, there have been several lines of research related to social development since Lorenz' (1935) identification of critical time periods for social bonding. Studies detailing early environmental effects on brain and behavioral development in numerous species appeared in the first decade of the 20th century. Here, I focus on several lines of earlier research in animal models that directly relate to the alterations in early social experiences described in our four cohorts, with particular attention to those preceding current research trends. These studies paved the road for modern neuroscience conceptualization on the how early experiences shape the social brain and forecasted recent findings on OT involvement in these processes. Furthermore, current terminology, including maternal proximity, maternal deprivation, peer rearing, and exclusive social bonds, as embedded within specific time windows and as exerting a lifelong impact on the adult animal, dates back to the 1950s and 1960s, as is the understanding that $\mathrm{CP}$ must be studied within a given species.
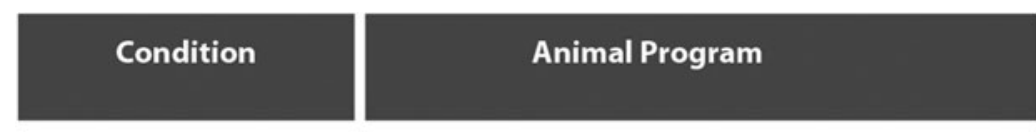

Prematurity

(Kangaroo Care)

Multiple Birth

Maternal Postpartum

Depression

Chronic Unpredictable Trauma
Maternal Proximity/Deprivation (Hofer, Denenberg) Handling (Levine) Social Buffering (Sullivan)

Peer Rearing (Suomi)

"Peers as Therapists" at the transition from Infancy to Childhood (Harlow and Suomi)

High/Low Licking and Grooming (Meaney)

Maternal Rotation (Denenberg)

Variable Foraging Demands (Rosenblum \& Paully, Coplan)
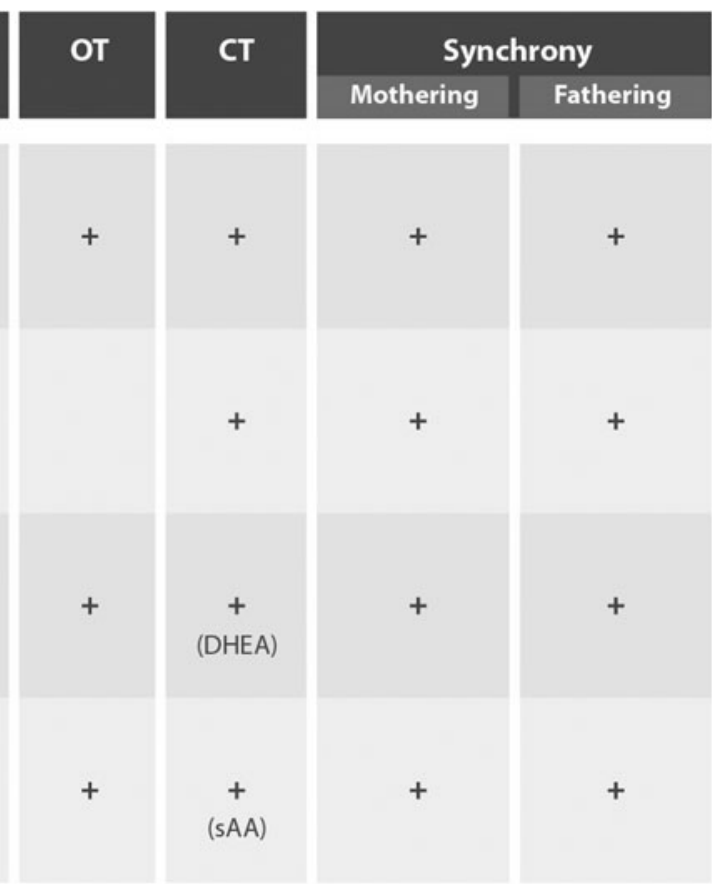

Figure 1. Four high-risk birth cohorts followed repeatedly from birth to 10 years. Each cohort involves a specific missing component from the early environment and utilizes a research program in animal models to generate hypotheses based on sensitive period conceptualization. Plus (+) signs for oxytocin (OT), cortisol (CT), and maternal and paternal synchrony represent available data in this domain for this cohort. DHEA, Dehydroepiandrosterone; sAA, salivary alpha amylase. 
Over 50 years ago, a review paper in Science (Scott, 1962), titled "Critical Periods in Behavioral Development," attested to the volume and importance of this line of work. Scott described a young lamb raised by him who later showed aberrant social functioning and was unable to bond with her ewe. The review clearly suggests the existence of $\mathrm{CP}$ that determine the direction of social, intellectual, and emotional development and antecedes research on the role of OT in selective bond formation of lambs and ewes (Keverne \& Kendrick, 1992), as well as between human mothers and infants. The review cites research beginning in 1904 that described specific time windows in prenatal life when embryos are most sensitive to the environment, forecasting prenatal programming research and highlighting interest in time windows of specific environmental effects over a century ago. Mechanisms related to social $\mathrm{CP}$ are defined as those that bring young animals into contact with conspecifics, such as infant smiles that elicit maternal care, and consistent with research on OT-DA connectivity, it is suggested that processes of primary socialization do not require external reward and are self-reinforcing in nature. Scott (1962) also notes disruptions during SP that resemble postpartum depression. The quick formation of a social bond in goats draws on the high emotional arousal caused by birth, and when birth fails to elicit high arousal, as in depression, bond formation is disrupted. In response, Schneirla and Rosenblatt (1963) describe their "social ontogeny" theory, which postulates that $\mathrm{CP}$ are hierarchical, with each stage setting the stage for the next by introducing changes in the female-litter relationship. Social ontogeny considers the fusion between maturational processes in the organism and the experiences it can draw from the environment and their combined effects on the organism's ability to meet evolutionary challenges and manage the stress response. Thus, in the early 1960s, Schneirla and Rosenblatt maintained that social ontogeny, the stage-by-stage effects of social environment on brain, can be understood (a) only within a given species and (b) only in relation to variations that alter experiences between mothers and infants.

Beginning in the 1970s and up to the present, most (or all) mechanisms described in these studies were found to be at least partially supported by the OT system, rendering this research particularly timely. These studies also provided the basis for our biobehavioral synchrony model (Feldman, 2007a, 2012a, 2013) by demonstrating that mammalian caregiving involves close attunement between maternal and infant physiology and behavior; that maternal proximity functions to regulate the infant's immature systems; and that the consistency of maternal-infant physical contact bears long-term impact on the adult animal. Most important, studies from this period indicated that for mammalian young, the coordination of physiology and behavior must be experienced within the nursing dyad during early SP (Hofer, 1970a, 1970b; Lorenz, 1950; Schneirla, 1946; Tinbergen, 1963). Of interest is that theories of social development dated to this period are the first to draw on animal research and incorporate SP notions, implicitly or explicitly. These include Bowlby's earlier work on the cross-generation transmission of attachment bonds (1953) and the nature of the infant's tie to his mother (1958), leading to the trilogy attachment, separation, and loss, but also Erickson's (1963) theory on socialization, which charts a sequence of CP each depicting a unique exchange of maturational processes and environmental challenges from lower to higher across the life span.

Several lines of early animal studies are particularly important to our four high-risk cohorts.

a. Touch, multiple attachments, peers, and the rearing environment: Among the most famous studies on early attachment is Harlow's research (1958) on infant monkeys' preference for a soft cloth figure rather than a feeding wire figure, which underscored touch as a critical component of attachment relationships. It is interesting that Harlow is also among the first to describe an infant's multiple attachments and their importance for later growth. In 1958, Harlow writes in his famous paper "The Nature of Love" that males are "endowed with all the essential equipment ... in one essential activity: the rearing of infants" (1958, p. 685). This preceded current interest in the neurobiology of fatherhood, research showing comparable levels of OT in mothers and fathers (Gordon, Zagoory-Sharon, Leckman, \& Feldman, 2010a), and studies indicating neural plasticity in fathers to accommodate the transition to parenthood (Abraham et al., 2014; Kim et al., 2014). Harlow also pioneered the study of peers as a distinct attachment bond. Harlow and Suomi (1971) noted that peers can serve as "therapists" when parenting is deficient, particularly at the transition from infancy to childhood, charting this transition as a unique SP in socialization. When 6-month-old isolated monkeys were introduced to 3-month-old normal peers, the exchange reversed much of the effects of maternal deprivation on social behavior, which was not the case for adult surrogates. This work forecasted the elegant research program of Suomi on peer rearing in rhesus macaques. While the peer bond was preferable to isolation, monkeys reared by peers were anxious and impulsive as adults, and showed aberrant stress physiology (Stevens, Leckman, Coplan, \& Suomi, 2009). Similar focus on the long-term effects of the social context is found in the work of Denenberg in the 1960s. Denenberg, Hudgens, and Zarrow (1964) compared mice reared by rats from birth and those fostered by rats after weaning with isolated and typically reared mice, and they found lasting effects of the rearing context and its timing on social preferences and aggression toward the other strain. This work anteceded research on how familiarity alters "empathic" behavior toward "out-group" in rodents (Bartal, Decety, \& Mason, 2011), or how OT response is transferred from the parent-child to the peer attachment at the transition from infancy to childhood (Feldman, Gordon, Influs, Gutbir, \& Ebstein, 2013).

b. Handling and social buffering: Another line of research dated to the 1950s involves the "handling" paradigm, the removal of young animals from the home cage for sev- 
eral moments daily during SP. The results of this line indicate that even seemingly minor alterations in early social experiences exert lifelong effects on physiology and behavior (Levine, 1956). Handling across the first days of life altered the adult animal's learning, emotionality, and arousal (Denenberg \& Bell, 1960). Animals handled for the first 20 days weighed more, whereas those handled for the first 10 days lived longer under conditions of food deprivation (Levine, Alpert, \& Lewis, 1957). Further, handling studies supported current work on the cross-generation transfer of physiological effects via alterations in the mother's early environment. Levine (1967) found that handled rats showed lower plasma CORT at weaning, and a similar effect was also found in nonhandled rats whose mothers were handled as infants, anteceding research on the cross-generational effects of high versus low licking and grooming (Cameron et al., 2008). This line of research ushered in work on "stress inoculation," the positive effects of moderate early stress on later resilience, by suggesting that mild activation of the stress system during SP enables more flexible functioning in later life (Daskalakis et al., 2013; Levine, 2005). We consistently found that some children exposed to continuous trauma or chronic maternal depression showed adaptive stress or OT response, and differences between more or less resilient children depended on the mother's stress hormones and OT functionality (Feldman, Vengrober, \& Ebstein, 2014; Pratt et al., 2015).

An interesting program stemming from handling research considers the "social buffering" effect of maternal presence on infant stress response during SP. This line of research charted a clear sequence of $\mathrm{CP}$ along specific time windows (Sullivan \& Holman, 2010). Neonatal rodents show an early period of hypoactive stress response on the first postnatal days when the HPA system is not yet functional (Latysheva \& Rayevsky, 2003). Following, there is a transitional period on Days 6-15, when infant stress response is functional but suppressed by maternal presence, and suppression of amygdala activity by maternal presence underpins this effect. After this transition period, the mature stress response is fully active, enabling infants to mobilize the stress response to face the world and its dangers. These studies informed current research on the effects of early maternal proximity on maturation of amygdala-prefrontal cortex (PFC) connectivity (Tottenham, 2014). Consistent with animal findings on social buffering, human studies demonstrate specific time windows in typical development when the child's amygdala response is suppressed by maternal presence, the elimination of this effect in orphanage-reared children, and the effects of such aberrant rearing conditions on long-term amygdala-PFC connectivity (Gee et al., 2013).

c. Maternal rotation and variable foraging demand: Research beginning in the 1960s considered the sequalae of inconsistent maternal care. Denenberg, Ottinger, and Stephens (1962) examined infant development when mothers rotated every day between litters from birth to Day 20. Infants of rotated mothers showed high levels of mortality, highlighting the centrality of consistent caregiving for mere survival. Another paradigm assessing inconsistent care is Rosenblum and Paully's (1984) "variable foraging demands" in bonnet macaque, which induced unpredictable caregiving due to changes in food availability from abundant food supply (low foraging demands) to conditions requiring mothers to spend much time foraging while tending to their infants (high foraging demand). Unpredictable conditions resulted in the worst outcomes. Compared to those in high or low foraging settings, infants in the variable condition showed lower sociality and greater withdrawal as juveniles and alterations to stress physiology in adulthood, including gene expression, low and flat cortisol reactivity, and impaired glucose metabolism (Coplan et al., 1996). Research reporting low and nonresponsive cortisol levels in children reared in extreme chaos (Tarullo \& Gunnar, 2006) or unpredictable war trauma (Feldman, Vengrober, Eidelman-Rothman, \& Zagoory-Sharon, 2013) is informed by such research. Inconsistent caregiving also resulted in lower expression of the OT receptor gene $(O X T R)$ in the hippocampus of infant rodents (Noonan et al., 1994), consistent with our findings on OXTR in war-exposed children (Feldman, Vengrober, et al., 2014).

d. Maternal proximity and hidden regulators: An important research program in animal models dated to the 1960s and 1970s is the work of Hofer on "hidden regulators" (Hofer, 1970a, 1970b). His program is the first to conceive the mother's physical presence as a total environment comprising a set of experiences embedded in the mother's body. In a series of elegant experiments Hofer $(1987,1995)$ separated and experimentally manipulated the discrete elements embedded in the mother's body, including milk, body warmth, smell, and movements, and showed that each component functions to regulate a specific biobehavioral system in the pup, such as heart rate, thermoregulation, growth hormone, or the stress response. This program contributed to our model on biobehavioral synchrony by demonstrating one-on-one bidirectional effects between specific physiological systems in mother and child via social contact. This work is also the first to show that maternal proximity during SP operates to downregulate physiological functions, which in absence of contact to mother's body will hyperactivate (Hofer \& Shair, 1987), thus anteceding molecular findings on the role of GABAergic inhibitory processes in the initiation and termination of CP (Takesian \& Hensch, 2013). Finally, long before OT became a well-known player in human social functions and was known solely for its role in birth and lactation, Hofer (1987) described in microlevel detail the temporal synchrony between mother and infant physiology and behavior as coordinated by the pulsatile release of OT during the first motherpup social interaction: the feeding context. 
Rat pups sleep throughout most of each nursing period . . a great portion of suckling occurred during sleep. . . . [T]he nutritive form, rhythmic sucking, was predominantly seen during the brief awakenings that occurred following milk let down. The mother rat releases oxytocin in pulses every 5-10 minutes . . produces a flow of milk into the tits. We found that rat pups almost always awaken to this milk let down, stretches, rhythmically sucks, and in $70 \%$ of the instances is back asleep within 45 seconds. To our surprise, although rat pups are awake $35 \%$ of the time while attached to their mother's nipple, for the periods before milk ejections, the rat pups were always asleep in the 15 seconds immediately prior to milk ejection and awake only about $10 \%$ of the time in the minute prior to milk ejection. Since milk ejections are evenly distributed throughout nursing episodes, this suggests a puzzling connection between sleep in the infant and milk ejection in the mother. Lincoln (1983) found that the periodic bursting discharge pattern of oxytocin-releasing neurons in the maternal hypothalamus was dependent on a threshold level of suckling stimulation by infants. . . Mother was always in slow-wave sleep at the time her milk ejection occurred. Like her pups, she wakes up after milk ejection. Taken together, these results tell a surprising story. The feeding transaction of the mother and infant are embedded in sleep. Infants sleep while they are nursing and suck while they are asleep. Their suckling induces milk ejection to occur in the mother, but she must also be in slow-wave sleep. Sleeping infants suck and are quiet behaviorally while asleep for twothirds of the typical nursing episodes thus producing just those conditions optimal for mother milk letdown. . . . Within a nursing episode, it is precisely those periods when the pups have all been quietly asleep that will provide the mother with the lack of disturbance that will in turn allow her to enter the slow-wave sleep state upon which her oxytocin release depends. (Hofer, 1987, pp. 642-643)

Thus, OT is placed on the developmental map as coordinator of bonding experiences via mechanism of synchrony during the first moments of social contact between mother and child.

\section{Biobehavioral Synchrony: Mechanism by Which SP Exert Their Effect}

Biobehavioral synchrony occurs during SP in social development and is the mechanism by which attachment bonds become selective and enduring, that is, dyad specific and long lasting (Feldman, 2007a, 2012a, 2012b, 2012c, 2013). Synchrony is a specific process within the general parental care constellation, is expressed during social contact between mammalian mothers and their young, and underpins the consolidation of the infant's affiliative neurobiology that later supports pair bonding and parenting in the next generation (Carter, 2014; Insel \& Young, 2001). In humans, in addition to experiences related to the maternal body and species-specific behaviors, synchrony involves finely tuned coordination between the social behavior of attachment partners, including mothers and fathers, lovers, and friends (Feldman, 2012a). At the behavioral level, synchrony describes the concordance of discrete behaviors that transmit social signals among conspecifics and the online organization of the two partners' behaviors into a unitary social event. The human social repertoire relies primarily on visuoaffective nonverbal cues, such as social gazing, facial expressions, or vocal tonality, but also in- cludes mammalian-general signals, such as affectionate touch, posture, and alterations in proximity position. Like songbirds, which must hear their species' song during a very narrow time window to become members of the peck (Margoliash, 2002), infants must experience synchronous coordination of their social behavior and affiliative biology during the SP time window, between birth and approximately 910 months. Unlike birds, this experience must occur within the "nursing dyad" and is not predetermined but contains great flexibility and is open to the immense personal, historical, and cultural variability of human societies. Following experiences with the attachment figure during SP, synchronous processes in humans occur between all members of the species, albeit to a lesser degree than with loved ones; for instance, humans synchronize social gaze while conversing with any social partner, familiar or stranger. This, according to Hofer (1987) and Rosenblatt and Lehrman (1963), is a critical aspect of being a mammal whose development begins with a period of dependence on maternal physical presence and its social inputs. Viewed from this angle, biobehavioral synchrony is a process most resembling the classic CP effect in humans. As demonstrated in the following, when the experience of synchrony is missing or altered during SP, there are marked deficits in children's social and regulatory outcome as well as in OT functionality and the stress response. Disruptions to other environment-sensitive systems are also observed, including sympathetic and parasympathetic functioning, sleep-wake rhythmicity, and executive control, indicating disruptions to brain stem mediated bottom-up and prefrontal top-down processes.

Biobehavioral synchrony is a critical mechanism in the evolution of mammals. Synchronous processes were described nearly a century ago by entomologists (Wheeler, 1928) as those by which a physiological response of one group member (e.g., neural firing or hormonal release) is coordinated with the behavior of another member (e.g., leg movements), leading to a four-pole biobehavioral matrix that ushers young members (e.g., a new ant) into the social group. This process enables a group of ants to jointly carry out a collaborative goal, such as carrying a grain of wheat to shelter, and is the mechanism conceptualized as providing the foundation for the capacity for social collaboration. In his recent book The Social Conquest of Earth, entomologist Ed Wilson (2012) suggests that such hypersociality that binds members of a multigenerational group into a social unit, which he terms eusociality, enabled ants to conquer the world of invertebrates and humans the world of mammals.

With the evolution of mammals, biobehavioral synchronous processes were integrated into the nursing dyad, and adaptation of young to their social milieu no longer occurred in the context of the group, like ants, fish, or birds, but within the intimacy of the nursing dyad and mother's body. Keverne (2013) highlights two events in the evolution of mammals leading to paradigmatic change in the brain, both involving mechanisms of synchrony. First, development of the placenta and the ensuing need for coordination of hypothalamic func- 
tioning in mother and fetus led to structural changes in the hypothalamus and thalamocortical networks that underpin human consciousness (Edelman, 2004), and imprinted a synchronous co-adapted function of maternal-infant physiology that was preserved across generations, a process in which OT plays a key role. Second, development of the neocortex transformed social skills from dependence on the bonding context into context-independent functionality. With the enlarged cortex, the attachment repertoire and its underlying neurobiology became independent from reproduction and projected onto symbolic and mental contexts. Whereas in small-brain mammals, mechanisms of preference, familiarity, reward, and stress reduction are behavior and olfactory based, in larger neocortex animals, mechanisms evolved to synchronize bonding-related processes with multiple brain systems and to coactivate hormonal priming with multiple cortical sites, particularly associative cortices. Such abilities prepare for life in a complex world, enable cultural variability, and link the neurobiology of affiliation with processes related to symbolic thought and complex sociocognitive skills. The interplay among CP, the OT system's role in supporting CP effects, and the role of biobehavioral synchrony as a mechanism by which the OT system can exert its effects during $\mathrm{CP}$ is presented in Figure 2.

Our research on biobehavioral synchrony provides support for these notions by demonstrating connections both between synchrony and the OT system in attachment contexts and between synchrony and context-independent sociocognitive brain networks and social skills unrelated to attachment, such as formal social abilities, symbolization, social reasoning, and empathy.

\section{Biobehavioral synchrony in parental attachment}

Similar to work on maternal-infant bonding in animals, research on face-to-face interactions as providing the context for synchronous coordination of maternal and infant's nonverbal signals in humans dates back to the 1970s (Brazelton, Koslowski, \& Main, 1974; Stern, 1977). More recently, it has been shown that such interactions also construct a platform for the online synchrony of physiological processes. For instance, during face-to-face interactions, mothers and infants coordinate their heart rhythms within lags of less than $1 \mathrm{~s}$; moreover, such biological synchrony was found to depend

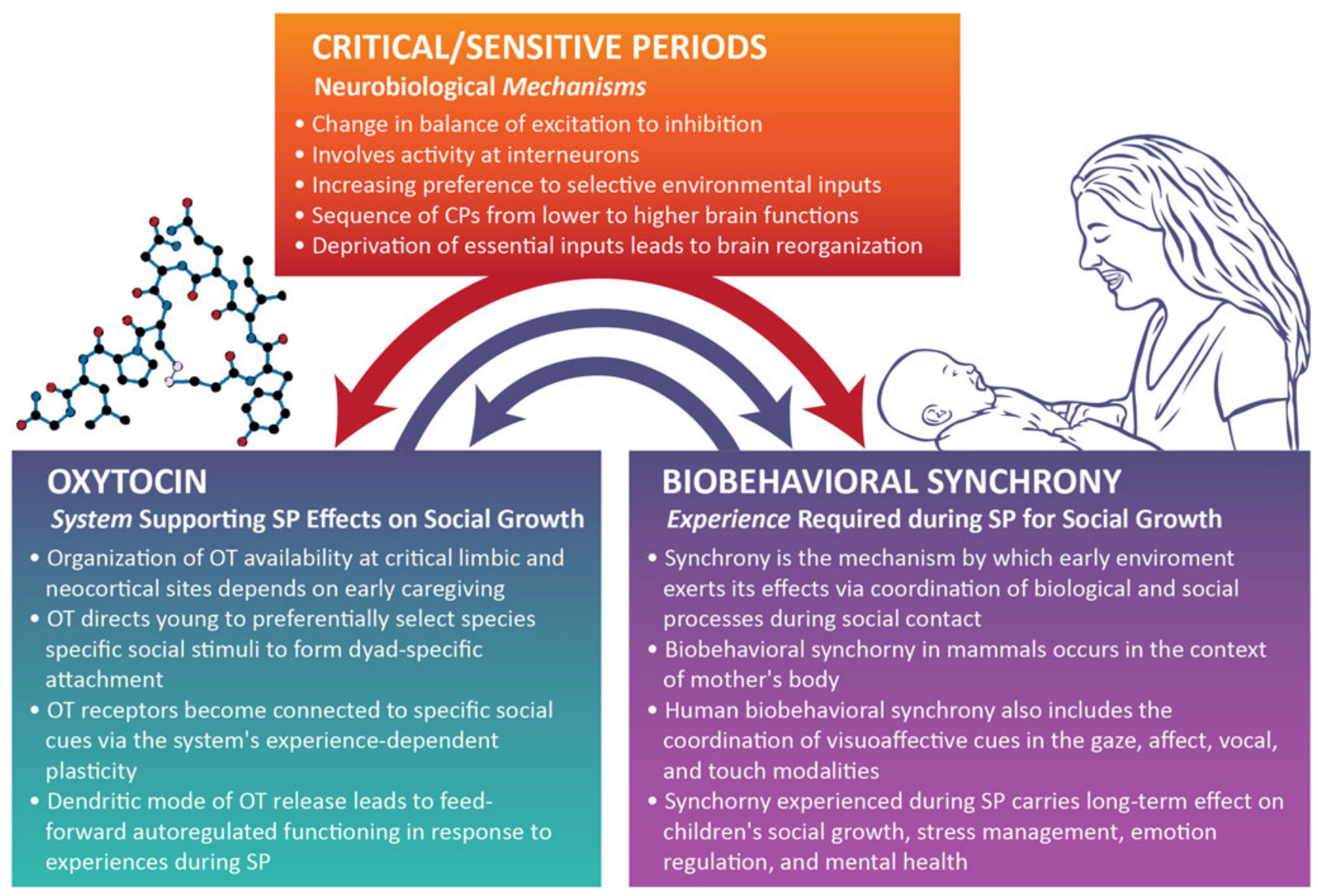

Figure 2. Critical periods (CP) involve specific neurobiological mechanisms observed across developmental domains (e.g., neuronal, physiological, sensory, motor). In the social domain, the oxytocin (OT) system employs these mechanisms to support social growth by utilizing processes of biobehavioral synchrony. The experience of synchrony during sensitive periods for social growth in turn shapes the infant's OT functionality across mammalian species. SP, Sensitive periods. 
on behavioral synchrony between mother and child. During moments of vocal and affect synchrony, mother-child heart-rate coupling was strong, whereas during nonsynchronous moments, no biological synchrony between maternal and infant heart rhythms was found (Feldman, Magori-Cohen, Galili, Singer, \& Louzoun, 2011). Similarly, during synchronous interactions, but not when mother was uncoordinated with the infant social signals, we found matched attenuation of the stress response in mother and child as well as synchronous alterations in respiratory sinus arrhythmia in response to social stressor (Feldman, Singer, \& Zagoory-Sharon, 2010).

One of the main requirements from a mechanism conceptualized as key in supporting human SP effects is to demonstrate longitudinal predictions between provisions during SP and outcomes over long epochs. In several low-risk cohorts followed from infancy to adolescence, we found that infant-mother and infant-father synchrony contributed in unique ways to social competencies. Whereas mother-infant synchrony focuses on the coordination of visoaffective social cues, father-child synchrony directs infants toward the environment, is more physical, and contains quick peaks of positive arousal (Feldman, 2003). Mother-infant synchrony at 3 and 9 months predicted self-regulated socialization at 2 years (Feldman, Greenbaum, \& Yirmiya, 1999), moral understanding and empathic dialogue in adolescence (Feldman, 2007c), and lower depressive, externalizing, and internalizing symptomatology in adolescence (Feldman, 2010). Synchrony with both parents predicted greater symbolic competence at 3 years, with different temporal patterns of symbolic expression with each parent (Feldman, 2007b). The maternal form of synchrony predicted greater social competence in kindergarten and the child's ability to engage in reciprocal positive activities with a best friend in adolescence. The father's form of synchrony predicted lower aggression in kindergarten and the teenager's ability to dialogue conflicts with best friends in a manner that included empathy and mutuality (Feldman, Bamberger, \& Kanat-Maymon, 2013). These outcomes can be tied back to the rhythmic-calm synchrony of mother and the tougher, physical synchrony with father.

Synchrony and the parental brain. Evidence that synchrony during SP is implemented in the brain is provided by research on the parental brain. In several studies, we found associations among brain activations, behavioral synchrony, and peripheral OT. While observing their infant's video, mothers and fathers synchronized their brain response online in a "mentalizing" network (Mar, 2011; Spunt, Satpute, \& Lieberman, 2011), including the temporal-parietal junction, inferior frontal gyrus, and medial PFC, and demonstrating "brain-to-brain coupling" of the maternal and paternal brain within attachment context (Atzil, Hendler, Zagoory-Sharon, Winetraub, \& Feldman, 2012). We theorized that brain synchrony played an important role in the evolution of human social groups and is an important mechanism by which group members, such as sports teams or medical crews, coordinate action within split seconds via synchronized physical activity. We further suggest that such coordination, critical for the survival of Homo sapiens, was critical in evolution of the human family, assisting mother and father to jointly care for their infant and maximize chances for survival.

Interactive synchrony is also related to maternal brain activations. Mothers who expressed more behavioral synchrony showed greater activation of the nucleus accumbens (NAcc), a key structure of the DA reward system, which was functionally connected to the sociocognitive circuit supporting parenting and to plasma OT. Thus, synchronous parenting provides underlying "reward" coloring to cortical processes implicated in parental care and binds the OT and DA systems in a brainbehavior link (Atzil, Hendler, \& Feldman, 2011). Finally, we tested maternal brain response to synchrony in others. Mothers underwent brain scanning while observing three mother-infant interactions: one synchronous mother and two nonsynchronous mothers, one depressed and one anxious, of children. During a home visit, mothers' own behavioral synchrony with her infant was videotaped. Synchronous interactions activated the brain reward system and increased activity in the NAcc and dorsal anterior cingulate cortex, a brain hub linked with reward and emotional understanding (Bernhardt \& Singer, 2012; Haber \& Knutson, 2010). Moreover, maternal synchrony with her infant correlated her dorsal anterior cingulate cortex response to synchrony in others. These findings point to mechanisms of "embodiment" and experience-dependent plasticity of the maternal brain: the more a mother engages in synchronous interactions, the greater her reward brain response to synchrony in others (Atzil, Hendler, \& Feldman, 2014).

Research on fathers' brains provides a model for experience-dependent brain plasticity not primed by hormones of pregnancy and childbirth. In a recent study (Abraham et al., 2014), we measured brain response, salivary OT, and parent-infant synchrony in three groups of first-time parents: primary caregiving heterosexual mothers, secondary caregiving heterosexual fathers, and primary caregiving homosexual fathers raising infants within a partnered relationship with no maternal involvement $(N=89)$. Parenting was supported by a general "parental caregiving" network, which was remarkably consistent across parents and integrated functioning of two networks: an emotional processing limbic-paralimbic network including structures implicated in vigilance, reward, and emotion processing (amygdala, ventral tegmental area, anterior insula, and anterior cingulate cortex), and a mentalizing network, including fronto-temporo-partietal areas (superior temportal sulcus [STS] and temporal poles) related to social understanding, cognitive empathy, and theory of mind. Mothers showed greater activations of the emotional processing network, particularly the amygdala, which was related to OT and synchrony, whereas fathers exhibited greater activation of the mentalizing network, particularly the STS, similarly linked with OT and synchrony, indicating distinct "maternal" and "paternal" pathways to the parental brain. Among primary-caregiving fathers amygdala activation was high similar to mothers, whereas STS activation was high 
similar to all fathers, with functional connectivity of amygdala and STS in this group, suggesting that the mentalizing system recruits the amygdala network in primary-caregiving fathers. Moreover, we found that in all fathers, the degree of amygdala-STS connectivity was related to the father's caregiving experiences: the more fathers were responsible for infant care, the greater STS-amygdala overlap that was observed. Because both the amygdala and the STS are key nodes of the social brain (Allison, Puce, \& McCarthy, 2000) and maintain an intrinsic interconnectivity (Harrison et al., 2009), these findings demonstrate for the first time brain plasticity in human fathers. Such plasticity during a $\mathrm{CP}$ for parent-infant bonding is observed in other biparental fathers (de Jong, Chauke, Harris, \& Saltzman, 2009; Kozorovitskiy, Hughes, Lee, \& Gould, 2006), where active involvement in caring for pups leads to functional connectivity between networks implicated in nurturance, learning, and emotionality in the paternal brain. Finally, gray matter increases were found in both mothers' (Kim et al., 2010) and fathers' (Kim et al., 2014) brains in key areas of the parental caregiving network and in accordance with the parent's social behavior, pointing to brain plasticity in the first months of parenting in areas linked with OT receptors and synchronous parental behavior.

OT and parent-infant synchrony. Numerous studies in our lab tested the involvement of OT in synchronous human parenting in low-risk families. Our first study followed a group of pregnant mothers from the first trimester of pregnancy to the first postpartum month. We found high individual stability in OT levels and OT during the first trimester predicted the expression of the maternal postpartum repertoire in human mothers, including the amount of mother gaze, affect, vocalization, and touch and its coordination with infant state. These findings confirm that, similar to other mammals (Nelson \& Panksepp, 1996), OT during pregnancy primes mothers for the expression of maternal behavior. The next study tested first-time mothers and fathers from birth and across the first 6 months of parenting. OT was found to be significantly higher in parents compared to nonattached singles. To our surprise, mothers and fathers showed comparable levels of plasma OT, indicating that fathers are biologically prepared for the parental role, and we found endocrine synchrony between husbands' and wives' OT levels. Parental OT correlated with the parent-specific repertoire: with social gaze, positive affect, and affectionate touch in mothers, and with stimulatory touch, exploratory focus, and positive arousal in fathers (Gordon, Zagoory-Sharon, Leckman, \& Feldman, 2010a). Triadic family synchrony during mother-fatherinfant interaction was predicted by maternal and paternal OT and by lower maternal diurnal cortisol, highlighting the links between the OT and HPA systems in early attachment (Gordon, Zagoory-Sharon, Leckman, \& Feldman, 2010b).

In several studies we manipulated OT production in the parents in a manner similar to animal research. In a touch manipulation study, 112 mothers and fathers (not couples) of 4- to 6-month-old infants engaged in the "play-and-touch" paradigm, a paradigm developed in our lab that asks parents to interact with their infant for $15 \mathrm{~min}$ in play that includes physical contact. Similar to the findings for the high versus low licking and grooming dams (Francis, Young, Meaney, $\&$ Insel, 2002), mothers who provided high levels of affectionate touch, but not those providing minimal contact, showed an OT increase following play. Similarly, fathers who engaged in high levels of stimulatory contact, but not those showing minimal contact, increased OT after play (Feldman, Gordon, Schneiderman, Weisman, \& ZagoorySharon, 2010). OT manipulation on parents was also tested in a within-subject OT administration study. Fathers were administered OT or placebo intranasally in a double-blind within-subject design while the infants were in a separate room. After $45 \mathrm{~min}$, the infant was brought back to the father and the two interacted in the face-to-face still face paradigm while respiratory sinus arrhythmia (RSA) was measured from father and infant, and hormones were assessed at multiple time points. OT administration to father dramatically increased the father's salivary OT, but the infant's salivary OT showed a parallel increase of about 30-fold without inhaling the substance. Under OT administration, fathers engaged in longer durations of gaze synchrony, touched infants for longer periods, and infants displayed more exploratory behavior. In parallel, OT administration not only increased the father's RSA, indicating greater autonomic readiness for social engagement, but also increased the infant's RSA (Weisman, Zagoory-Sharon, \& Feldman, 2012). Cortisol levels of father and infant in response to the still-face were related to father-infant synchrony (Weisman, Zagoory-Sharon, et al., 2013), and father's testosterone, which is typically associated with lower paternal engagement, declined under OT administration, but only in synchronous fathers (Weisman, ZagoorySharon, \& Feldman, 2014). Using complex computational models, we found that OT impacted subtle motion cues in parent and child, such as head proximity, velocity, and movement, in patterns resembling birds' social repertoire (Weisman, Zagoory-Sharon, et al., 2013). These findings clearly demonstrate coordination of biology and behavior during social contact and their effect on multiple hormonal systems (Weisman \& Feldman, 2013).

OT provides an integrative platform for a host of hormones implicated in parental care (Feldman, 2012a). Interacting with their 6-month-old infants, fathers' OT and prolactin (PRL), two hormones underpinning fathering in biparental mammals (Wynne-Edwards, 2001), were interrelated. Whereas paternal OT correlated with father's attachment behavior during free play, PRL was associated with behavior during toy exploration and father's support of exploratory play. OT and AVP were measured in 120 mothers and fathers of 4- to 6-month-old infants. OT and AVP showed low but significant correlations and were each associated with a distinct behavioral repertoire. Parents with higher OT exhibited more affectionate touch and responded to infant social bids by increasing social saliencepositive affect, social gaze, or affective touch. In contrast, par- 
ents with higher AVP provided more stimulatory touch and responded by increasing object salience-object presentation and joint attention to objects (Apter-Levi, Zagoory-Sharon, \& Feldman, 2014). Testing biomarkers of the affilation (OT and AVP), reward (beta endorphin), and immune (interleukin-6) systems in new parents, new lovers, and unattached singles, we found an increase in each hormone during periods of bond formation. In both new parents and new lovers, indices of the affiliation, reward, and immune systems not only increased in levels but also became interconnected. Whereas no correlations were found amond OT, beta endorphin, and interleukin- 6 among singles, the three were interrelated in parents and lovers (Yaniv et al., 2014). Such findings are consistent with animal research pointing to OT-DA links following birth (Shahrokh et al., 2010) and OT effects on the immune system mediated by social behavior in parent-child and marital relationships (Carter, Altemus, \& Chrousos, 2001; Gouin et al., 2010; Smith \& Wang, 2012). Finally, the combined effects of OT and testosterone, linked with mate selection and decreasing in fathers, on maternal and paternal care was tested. In mothers, OT predicted more synchrony, but only in the context of high testosterone. Among fathers, two separate effects emerged: positive for OT, and negative for testosterone. These findings suggest that OT cross-talks with other endocrine biomarkers to support human parental care (Gordon, Pratt, Zagoory-Sharon, \& Feldman, 2015).

Large-cohorts studies tested the distribution of OT in humans and its relation to genetic variability. In a large sample of women and men $(N=598)$, higher OT was associated with greater attachment anxiety in women and lower trait anxiety in men, indicating gender dimorphic links possibly related to the evolutionary-based involvement of fear and vigilance mechanisms in maternal bonding (Weisman, ZagoorySharon, et al., 2013). In a second study $(N=352)$, plasma OT was associated with allelic variations on the OXTR and cluster of diffentiation 38 (CD38) genes that index greater functionality of the brain OT system, attesting to relations between central and peripheral indices of OT in humans (Feldman et al., 2012). Both genetic and peripheral markers of OT correlated with higher frequencies of parental touch, longer durations of social gaze synchrony, and memories of better parental care in childhood.

Prospective research on the cross-generation transmission of affiliative biology from parents to child and from parental to filial (friendship) attachment is essential for demonstrating human SP effects and was tested in two studies. Parents and infants engaging in the "play-and-touch" paradigm during SP for the maturation of the OT system (4-6 months) showed parallel increases in salivary OT from baseline to postplay moderated by behavioral synchrony. Among dyads with high behavioral synchrony, close correlation was found between parental and infant OT response, but among nonsynchronous dyads, no biological synchrony emerged (Feldman, Gordon, \& Zagoory-Sharon, 2010). These findings support our main hypothesis: that the parent's physiology can externally regulate the infant's physiology during SP for social growth through relational behavior during moments of social contact. Finally, in a longitudinal study, we examined outcomes of OT and synchronous parenting during early SP on the child's OT response in the next attachment bond with their "best friends" at the transition from infancy to childhood. Parent-child interactions and parental OT were measured at 1 and 6 months, and parental plasma OT and genetic variability on the multiple single nucleotide polymophisms (SNPs) on the OXTR and CD38 genes were assessed and combined into a single index. At 3 years, children interacted with their first "best friend" in the home ecology (a setting comparable to the play of juvenile animals in their natural habitat) and salivary OT was measured. Parental OT during the first 6 months of parenting interacted with parental behavior to predict the child's OT response and social reciprocity in the next attachment, indicating that social experiences within the parent-infant bond during SP carry long-term effect on the child's affiliative biology and his or her ability to form the next bond with individuals outside the family system (Feldman, Gordon, et al., 2013).

Romantic attachment. The results on romantic attachment are presented to demonstrate that similar process support parental and romantic attachment at both the endocrine and the behavioral levels. Research in animal models, particularly in prairie voles, demonstrates the critical involvement of OT in pairbond formation in mammals (Ross \& Young, 2009). We recruited 120 young adults (60 couples) who began a romantic relationship within the last 3 months to address the biological and behavioral changes that occur during the period of falling in love and compared these couples to unattached singles. Plasma samples of multiple hormones, diurnal cortisol patterns, and couple interactions in positive, conflict, and support-giving paradigms were assessed. Two-thirds of the couples stayed together 9 months after the relationship began and were seen again. We found that OT levels showed marked increase at the initiation of romantic love, and levels were higher even in comparison with new parents, highlighting the period of human pair bonding as one eliciting the greatest OT response across the life span. OT levels were individually stable across the 9-month period and, similar to parenting, correlated with social synchrony between partners during positive encounters, including expressed positive affect, matched dyadic states, affectionate touch, and mutual gazing (Schneiderman, Zagoory-Sharon, Leckman, \& Feldman, 2012). Cumulative risk on the OXTR, a combined index of five SNPs associated with risk for disorders of social functioning, was associated with diminished capacity to provide empathy to the partner's distress during a "support giving" paradigm (Schneiderman, Kanat-Maymon, Ebstein, \& Feldman, 2013). We next assessed OT in relation to five hormones implicated in affiliation (AVP, PRL, and testosterone) and stress (cortisol and DHEA) and their associations with the couple's behavior during conflict discussion, particularly the dimensions of empathy and hostility, which are individually stable behaviors that predict marital dissolution in longitudinal research (Gottman, 1998). Consistent with the bio- 
behavioral synchrony model, we tested mutual influences of hormones and behavior among partners during the SP of pair-bond formation. The results revealed intriguing biobehavioral links. The stress hormones cortisol and DHEA each had a direct effect on hostility: the higher their levels in the individual, the more hostility that person expressed during conflict discussion. Cortisol and testosterone had a combined actor-partner effect: cortisol predicted lower empathy, but only when the partner also had high cortisol, not when the partner had low cortisol. Similarly, testosterone had a combined effect: an individual's testosterone predicted high hostility only in the context of high partner's testosterone. The results related to OT were of particular interest: OT was the only hormone to have a direct partner effect: the higher the partner's OT, not the individual's OT, the more that individual expressed empathy toward the partner. Low empathy in turn was the factor predicting the consolidation or dissolution of the bond and led to relationship breakup 9 months later (Schneiderman, Kanat-Maymon, Zagoory-Sharon, \& Feldman, 2014), highlighting synchrony of biology and behavior as mediated by the OT system, which binds partners into an affiliative bond.

Overall, these studies clearly demonstrate the involvement of OT in human bond formation across the three forms of bonding in mammals: parental, pair, and filial (close friends). The common thread among these bonds is their biobehavioral nature. The formation of a bond, like entry into a $\mathrm{CP}$, involves cascade of physiological processes supported by OT and the fine-tuning of dyadic behavior based on familiarity with the partner's pace and rhythms, signals and pauses, tastes and sensibilities.

\section{OT, SP, and Experience-Dependent Plasticity}

Over the past decade, OT has been implicated in nearly every social ability of the human adult, including empathy, trust, stress management, social memory, and emotional face recognition (Meyer-Lindenberg, Domes, Kirsch, \& Heinrichs, 2011). These findings provide ample evidence for our central proposition: as mammals, humans master social competencies via the system that underpins bonding during its SP, and maturation of the social brain occurs in the context of the nursing dyad. OT induces a physiological state of quiescence that affords participation in the world without fear and stimulates the desire for social contact (Burgdorf \& Panksepp, 2006; Carter, 1998).

\section{Timing is critical for OT functioning around birth}

For the definition of a SP effect, timing is critical. Research in animal models underscores the importance of timing in OT activity, particularly in the period surrounding birth, and describes mechanisms of both inhibition and facilitation in these effects. During pregnancy, the maternal brain must protect the fetus from adverse programing by maternal glucocorticoids and facilitate timely and safe birth. In late preg- nancy, K-opioids, coproduced by OT neurons, inhibit OT release in the posterior pituitary to prevent premature labor. The inhibition of opioids production decreases at the end of pregnancy, enabling action-potential trigger of OT release during partuition. This involves major reorganization of the OT system during labor, leading not only to OT release from magnocellular neurons in the hypothalamus but also to dendritic release through binding of OT autoreceptors and coordinated burst firing. OT pulsatile release from the posterior lobe is controlled by opioid-based mechanisms, which can coordinate birth according to favorable environmental conditions, charting the first integration of environmental events and OT in the life of the newborn (Brunton \& Russell, 2008). OT during birth causes GABA signaling to change from excitatory to inhibitory, and the surge of OT at birth enables synchrony of fetal hippocampal neurons and the transition from prenatal to postnatal life (Bali \& Kovacs, 2003; Blyth, Hauger, Purdy, \& Amico, 2000). Reorganization of the OT system following childbirth also occurs in the mother, contingent upon infant stimuli. Numan and colleagues (Numan, 2006; Numan et al., 2005) found that OT release in the maternal brain is similar in response to suckling and OT administration and functions to sensitize a limbic network related to reward and emotionality, including the ventral tegmental area, NAcc, insular cortex, hypothalamus, and amygdala, structures similarly implicated in the human parental care network. These serve to imbue the infant with reward value and are critical for emotional development, survival, and motivation (Sokolowski \& Corbin, 2012). The transition to motherhood involves increased connectivity between the medial hypothalamus, particularly the OT-primed medial preoptic area, and the amygdala, which serves to inhibit maternal aversion to infant cues. Thus, the initiation of the maternal SP, which sets the initiation of maternal care, involves a two-stage process: depression of the typical avoidance response of female rodents to infant stimuli and consolidating the reward from her own infant through excitation of dopamingergic pathways and increasing OT-DA connectivity.

Receptors for OT are found in the fetal brain, and the prenatal development of OT is activity dependent, suggesting SP effect (Lee, Brady, Shapiro, Dorsa, \& Koenig, 2007). Hammock (2014) notes that during the time of weaning, OT functioning in the infant's brain is similar to that of the adult's and follows the species-specific pattern in binding sites for receptors that are critical for maturation of the social brain. Peak OXTR ligand binding in the mouse neocortex occurs during the perinatal period and at that time the system is particularly open to social inputs. In rodents, olfactory imprinting occurs at this period, enabling the infant to navigate the social world. Variability in OT availability supports the acquisition of social experiences during the SP via contact with mother and conspecifics. For instance, $O X T$ knockout mice show a decrease in distress vocalization (Liu et al., 2008), leading to less contact with mother and impoverished social experiences. This is similar to findings from premature infants who cry less (Johnston, Stevens, Craig, \& Grunau, 1993), 
are deprived of the OT-enhancing experience of breastfeeding, and continue to receive less maternal investment across infancy and childhood (Feldman, Rosenthal, et al, 2014). Thus, already at birth OT shapes the infant's attachment repertoire, which triggers parallel behaviors in the mother and in turn enhances OT functionality in the child. In primates, OT in cerebrospinal fluid was higher in mother-reared than peer-reared infants (Winslow, Noble, Lyons, Sterk, \& Insel, 2003) and maternal behavior correlated with greater plasma OT (Maestripieri et al., 2009). Skin-to-skin contact increased OT in rodents' hypothalamus via biobehavioral synchrony. Kojima, Stewart, Demas, and Alberts (2012) placed mothers and infant rats in skin-to-skin contact and OT was measured from serum and in the hypothalamus during contact and following 1-hr, 2-hr, and longer separation. At baseline, no correlations were found between OT in the brain and the periphery, but after an hour of separation, levels were synchronized. Among infant receiving contact, central-peripheral coordination remained, but no associations were found among those in isolation, highlighting the role of maternal contact for brainperiphery synchrony of OT production.

SP effects are also noted in research showing long-term impact of OT manipulations after birth, particularly on males. Postbirth OT administration is suggested to function as a hormonal imprinting mechanism and to affect receptors distribution of OT and AVP, as well as DA and serotonin metabolism in the adult animal (Carter, 2014). Evidence indicates imprinting-like effects of exogenously or endogenously stimulated OT. Bales and Carter (2003) showed that a single dose of OT $24 \mathrm{hr}$ after birth increased partner preference in adults, and while single administration improved partner preference, chronic administration reduced it. In parallel, human studies showed long-term negative effects of birth induction by Pitocin (synthetic OT) particularly on males (Dahlen et al., 2013). Environmental enrichment after birth increased mRNA OT in the paraventricular nucleus (PVN), and greater frequencies of OT were observed on MESPEC in sensory and visual cortices, indicating that sensory experience alter the production of OT in the PVN and its availability in the cortex (Zheng et al., 2014). The early licking and grooming effect of mothers increased methylation of estrogen alpha in the medial preoptic area, and the effect was maintained in children (Champagne et al., 2006). This cross-generation transfer had a SP of P6-P10 in rats, which corresponds to the first month in humans.

\section{OT pulsatility and SP}

The pulsatile nature of OT release reflects a burst-firing pattern of magnocelular neurons, and the synchrony of bursting neurons in the PVN and the supraoptic nucleus (SON), the two major sites of OT production in the hypothalamus, facilitates plasticity (Lightman et al., 2001; Uvnas-Moberg, 1997). The rhythmic nature of OT activity supports maturation of circadian rhythms and enables homeostasis, thermoregulation, and energy preservation, connection of OT with cholecystokinin, glutamate, and GABA, and critical effects on regulating the HPA stress system (Theodosis, Montagnese, Rodriguez, Vincent, \& Poulain, 1986). In invertebrates, pulsatile release underpins homeostatic functions such as thermoregulation, cardiovascular control, and hormonal release. With the evolution of mammals, pulsatililty was incorporated into the birth context (Goodson, Kelly, \& Kingsbury, 2012); yet its ancient connectivity with brain stem mediated functions enables OT to promote sympathetic arousal without inhibition, what Carter and Porges (2013) call "immobility without fear," unique physiological state that promotes approach behavior and buttresses the unique combination of inner arousal and sense of safety that defines "love." Nelson and Panksepp (1966) suggest that the pulsatile release of OT, its surge and inhibition during play, provides the biological platform for the formation of social preferences that enable navigation in the social world.

Despite the importance of OT pulsatility to multiple physiological functions, it is still unclear whether the rhythmicity of hypothalamic hormones has physiological relevance for the infant (Hammock, 2014). Much research is needed to understand how OT functions within the infant's brain or how it programs the experience-dependent maturation of social brain networks. Pulsatile OT released during labor and nursing reduces glia around OT cells in the SON and PVN, which places OT neurons in greater proximity to each other and promotes greater functionality of the system. OT in the infant's blood already shows indication of pulsatile release (Marchini \& Stock, 1996). Pulsatile activity is particularly important for social imprinting during the SP time window for altricial mammals, a time when young learn how to be their own species and acquire the critical multisensory cues that imply being a member of that species. Cushing and Carter (2000) injected OT in pulsatile and nonpulsatile ways and found that only the pulsatile administration caused preference to the partners to whom females were exposed, indicating that pulsatility supports the imprinting function of OT. OT receptors are spread throughout the body (the heart, pancreas, gut, and sexual organs), and the pulsatile release from far sites enables this powerful molecule to be highly open to environmental effects and epigenetic programming (Carter, 2014). Thus, the pulsatile nature of OT release appears to play an important role in the plasticity function of OT and its SP effects.

\section{OT and experience-dependent plasticity}

Recent research has implicated OT in processes of experience-dependent plasticity not only in social-related brain structures but also in brain areas unrelated to sociality that support sensory and cognitive processes, including crossmodel perception, recognition, and memory. OT is implicated in plasticity at the cellular level and plays a key role in augmenting plasticity and specificity within various structures as well as binding unique pathways between structures. In an elegant study, Zheng et al. (2014) induced deprivation by either whisker trimming or isolation and dark rearing. 
On Day 14, pyramidal neurons in sensory cortices showed less spontaneous firing and less excitatory synaptic transmission in addition to less OT firing from the PVN and lower OT availability in neocortex. Both in vivo OT injection to the neocortex and sensory enrichment rescued the effect of deprivation on cross-modal plasticity, underscoring the role of OT in promoting cross-modal, experience-dependent plasticity. This study also links OT with the associative cortex and charts pathways for its involvement in processes outside the immediate social bond.

Another line considers the role of OT in plasticity related to social recognition memory. Gur, Tendler, and Wagner (2014) examined the role of OT in acquisition of long-term social recognition memory through connectivity between the olfactory system and the medial amygdala (MeA) and described the role of OT in memory consolidation via mechanisms of synaptic long-term depression. Some molecular processes that underpin the formation of long-term social memories, such as protein synthesis, occur in the MeA, and these are augmented by activity of OT neurons. The enhancement of social recognition through OT activity was achieved via inhibitory mechanisms. Exogenous OT administration increased long-term depression in the olfactory-MeA pathway, whereas OT antagonist inhibited this process. It appears that at least one process in the acquisition of long-term social memory involves depression of specific synaptic contact in the olfactory-MeA pathway, and familiarity with social stimulus involves depression of inputs through the olfactory system. This implies that plasticity at the molecular level requires first depression of general input and then activation of a specific synaptic connection. Several lines of evidence suggest that the prosocial effects of OT in mammals include, as a first step, reduction of response to aversive social stimuli, which are detected by the olfactory system, and then increase in network connectivity to enable response specificity to a social target (Bosch, Meddle, Beiderbeck, Douglas, \& Neumann, 2005; Bosch, Waldherr, Nai, Young, \& Neumann, 2006). OT acts to attenuate aversive olfactory responses at several stages of the process. Although olfactory cues are less central to human bonding, the same limbic system, including the hypothalamus, NAcc, bed nucleus of stria terminalis, lateral septum, and MeA, supports human social behavior and OT functions as a modulator of neural activity within this system. Sokolowski and Corbin (2012) suggest that humans are wired for social behavior via activity of this limbic circuitry, which regulates critical behaviors related to survival and motivation in the service of social bonding. One important role of OT is its role in long-term depression in the amygdala. This enables attenuation of amygdalar response to aversive social stimuli in order to promote social contact during SP and facilitate bond formation.

Another form of plasticity involves the increase of signal to noise ratio in the hippocampal interneurons. Owen et al. (2013) showed that OT enhances cortical information transfer while lowering background activity, thus improving signal to noise ratio. OT not only increased fast-spiking interneurons activity but also sharpened spike timing and reduced postsynaptic excitatory current in pyramidal cells. This may provide insights on how a diffusely delivered neuromadulator, such as OT, can improve performance of a neural circuitry that requires synapse specificity and millisecond precision. By selectively modulating inhibitory neuron firing and playing a role in synaptic depression, OT can regulate signal to noise in both the hippocampus and the amygdala.

Finally, OT is implicated in plasticity of the brain reward system (Dölen, Darvishzadeh, Huang, \& Malenka, 2013). In mice, OT acts as a social reinforcer within the NAcc core via presynaptic long-term depression of excitatory transmission in medium spiny neurons. The NAcc receives OT receptors containing inputs from multiple regions, and genetic deletion of these receptors abolished the reinforcing properties of social interactions, implicating OT in reward-related plasticity.

\section{Dendritic release and autoregulated activity}

A unique feature of OT in the brain is its releases from OTproducing sites in the PVN and SON, two hypothalamic nuclei, as well as from dendrites. Thus, OT can operate at very far locations and have long-lasting effects on behavior, particularly on sites with receptors for OT but no OT-containing projections. To address the role of OT in experience-dependent plasticity, it is important to understand how a diffuse signal can have specific effects on behavior. Emerging evidence suggests that peptides have a special role in information transfer, which differs from classic neurotransmitters (Pert, Ruff, Weber, \& Herkenham, 1985). Neuropeptides can prime vesicle stores for activity-dependent release, and this enables temporary functional reorganization of neural networks. Dendrites are a central source of peptide release, and this release is diffuse in nature, not directed toward the synapse, and maintains long half-life in the central nervous system and extracellular fluid (Ludwig \& Leng, 2006). OT can diffuse to far targets where it can lead to reorganization of neural networks that impact stress, immune functions, reward salience, and brain stem mediated autonomic processes.

Neurons typically communicate by a small number of neurotransmitters, which act with spatial and temporal precision, are stored in terminals, release mainly in synapses, have halflife of about $5 \mathrm{~ms}$, and are limited to the synapse with great efficiency. Unlike neurotransmitters, peptides can be stored in large vesicles that respond to electrical activity, are not limited spatially by synapses or temporally by quick degradation, and can release from anywhere in the neuron: axon, soma, or dendrite. This enables peptides not only to have far-reaching effects but also to incorporate experiences occurring during SP into an autoregulated function that preserves the conditioned state and initiates OT release to experience learned during SP.

Mechanisms of synchrony and pulsatility are central for OT functionality. The OT-AVP system is synthesized by several thousand large neurons (magnocellular neurons) whose cell body is mainly located in the SON and the PVN nuclei 
in the hypothalamus. There are also OT-producing parvocellular neurons in the PVN, which project to the brain stem and spinal cord, and support homeostatic functions, but their role in social processes is still unclear. Magnocellular neurons also release OT through their dendrites, which regulate OT release to the bloodstream. Dendrites involve $80 \%$ of the surface of neurons and are a central source of peptide release within the brain (Gimpl \& Fahrenholz, 2001). For dendrite release, pulsatility is critical. Pow and Morris (1989) found that if a neuron releases peptides from its axon terminal, it releases simultaneously from its dendrite, and such synchronous release is essential for the peptide's role in interneuron communication and quick processing.

The uniqueness of dendrite secretion involves the capacity for autoregulated activity that is primed by experience during SP. Dendrite and axonal modes of release are regulated by different processes. Several chemical signals, including OT, can cause dendrite release without increasing electrical activity, and through peptide feedback this release can be selfsustaining and therefore long-lasting. Such release creates an independent mechanism of feedforward that is based on experience and causes its own OT release. Signals that cause mobilization of intracellular calcium stores can prime dendrite peptide stores to be available for activity-dependent release. This leads to autoregulation (feedback to the original cell to produce more OT). OT functions at both pre- and postsynapsis to attenuate GABA, enlarging the extracellular interactions between OT cells while at the same time reducing interactions with other stimuli, a process leading to synchronization in time and across multiple areas in the brain. Once activated, dendritic OT release can be self-sustaining and functions as positive-feedback to activate repeated busts. This is how social experience during SP can impact the nature of OT release, the stimuli that will trigger it, and its ultimate organization in specific sites in the brain. Thus, while dendritic peptide release is typically not activated by electrical spikes, special conditioned signals can triggers OT dendrite release without electrical activity. This relocates OT in vesicles from reserve to stores that are releasable and makes OT available for activity-dependent release for long-term effects. Release is already shaped by the primed behavior experienced during SP, and parenting provides the best example of such priming of the infant OT. According to Ludwig and Leng (2006), this quality of peptide dendritic release enables a very crude administration of OT without temporal or spatial sophistication to cause coherent functional effects on behavior that are long-lasting and self-sustaining. While neurotransmitters transmit information from one neuron to another, peptides transmit information between populations of neurons and can act across great distances. Thus, priming of experiences during SP is the mechanism by which a diffuse signal releasing OT, such as interactive synchrony, can cause coherent, long-lasting consequences.

Overall, the nature of its far-reaching activity and the specific mode of release link the OT system with recent research on $\mathrm{CP}$ at the molecular level. The initiation of a CP (a) in- volves alterations of the balance between excitatory and inhibitory processes and (b) implicates functioning in interneurons (Hensch, 2005). As seen, OT plays a key role in plasticity in both the excitation to inhibition balance and interneuron functioning. Further, OT is critical in modifying the excitation to inhibition balance by causing GABA signaling to change from excitatory to inhibitory during birth. This points to the important, yet to be fully described, role of OT in opening a time window of increased sensitivity in the brain to specific environmental experiences that marks a SP.

\section{Four High-Risk Cohorts From Birth to 10: Unique Windows to SP in Humans}

Our four birth cohorts, followed from birth to 10 years with the preterm cohort currently seen in young adulthood (1819 years), each represent a different angle on how children grow with a specific critical element missing from their early environment during SP. At the same time, the results from these repeatedly assessed cohorts also demonstrate the complexity and multifinality of the human condition. The human brain and human parenting are plastic and malleable, and we find remarkable adaptation in later life in some cases. Human infants are extremely creative in utilizing resources in their ecology for growth after initial deprivation, but all these "resources" involve activation of meaningful attachment relationships. This can be seen in children's use of sensitive fathers in the case of chronically depressed mothers, families using social support networks in the case of lengthy war exposure, or children using the sibling relationship in the case of infants born as part of a triplet set. In addition, much reparation effects are observed after infancy, particularly at the transition from infancy to childhood (2-5 years), a period when monkeys raised in isolation can use peers to counteract the effects of early deprivation (Harlow \& Suomi, 1971). These findings clearly demonstrate that in order to advance understanding on how initial conditions shape human development, it is essential to follow children longitudinally, well beyond the early SP of infancy, assess periods of developmental arrest and regrowth, and describe how new environmental provisions contribute to continuous maturation of brain systems in promoting resilience. An overview of the four cohorts and the effects of each condition on children's hormonal, cognitive, social, emotional, and mental health outcomes across sequential SP appear in Figure 3.

\section{Longitudinal Cohort I: Premature infants and the Kangaroo Care intervention}

Extant research, including our own, indicates that premature infants, due to both immature brain and maternal deprivation during the SP for maternal physical contact, lag behind their full-term peers on a range of physiological, cognitive, social, emotional, and self-regulatory outcomes (Feldman \& Eidelman, 2003, 2007a, 2009a). In support of the SP approach, three studies from our lab following preterm infants from 


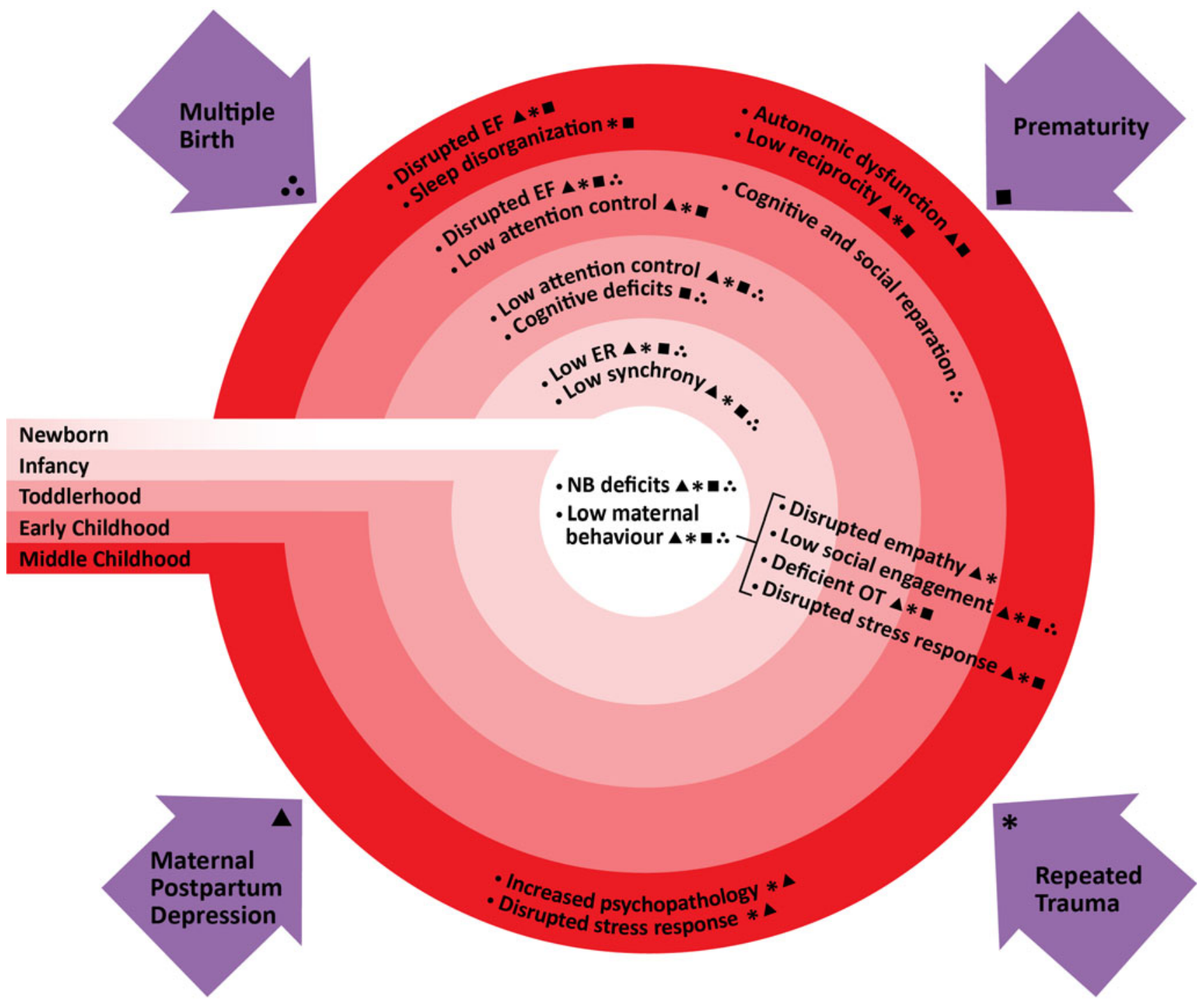

Figure 3. Four high-risk birth cohorts observed repeatedly from birth to 10 years including prematurity (square), multiple birth (three dots), repeated trauma (asterisk), and maternal postpartum depression (triangle). Deficits in maternal behavior and neurobehavioral competencies in the neonatal period observed across cohorts led to cohort-specific developmental difficulties across domains. Low maternal postpartum behavior predicted disruptions to hormonal (oxytocin [OT] and stress response) and social-emotional outcomes from infancy to middle childhood. Among healthy triplets, cognitive and social reparation was observed in early and middle childhood. NB, Neurobehavioral; EF, executive function.

birth indicated that the intactness of regulatory systems at birth (particularly systems that mature in mammals in the context of the mother's body, such as heart rate and circadian rhythms) play a critical role in supporting age-appropriate regulatory functions until 5 years of age. Outcomes disrupted by dysfunction of heart rhythms and the biological clock in the neonatal period included emotion regulation capacities across the first year, attention regulation skills during the second year, and emerging self-regulatory capacities at the transition from infancy to childhood (Feldman, 2009). In a second study, neonatal sleep states were observed in 10-s epochs across four consecutive evening hours, and mathematical modeling defined three groups with no differences in medical or demographic conditions (Weisman, Magori-Cohen, Louzoun, Eidelman, \& Feldman, 2011). Infants whose sleep-state transitions were characterized by shifts between quiet sleep and alert wakefulness exhibited the most optimal development, including greater neonatal neuromaturation, less negative emotionality in infancy, better cognitive development from 6 to 24 months, and more optimal verbal, symbolic, and executive competencies at 5 years. In contrast, infants who cycled mainly between states of high arousal, such as active sleep and cry, or between short episodes of active and quiet sleep showed poorer outcomes. These findings indicate that a more optimal balance between excitation and inhibition in the neonatal period provides an index of system maturity and predicts a better outcome, consistent with $\mathrm{CP}$ notions on the balance of excitatory and inhibitory mechanisms. Finally, we followed premature infants from birth to 10 years at seven time points (birth; $3,6,12$, and 24 months; 
and 5 and 10 years), and at each stage we observed parentchild synchrony and emotion regulatory skills using ageappropriate behavioral paradigms. A longitudinal autoregressive structural model indicated that, consistent with the prediction of the biobehavioral synchrony model, synchronous parenting and child regulatory capacities were mutually influencing over time: more synchronous parenting at one time point led to greater regulatory capacities at the next stage and vice versa. As predicted by the dynamic systems model (Fogel, 1993; Thelen \& Smith, 1994), the tightness of longitudinal effects increased as children grew and systems became more constrained and self-perpetuating. Consistent with the dynamic systems model, we suggest that this field of mutual influences (between synchronous parenting and regulatory structures of brain and behavior) is the field that shapes social outcomes across multiple domains and long epochs, including the capacity for empathy, health behavior and accident proneness, behavior problems, and autonomic functions, buttressing organization of the social brain (Feldman, 2015).

Beyond research linking early maternal deprivation with low regulatory capacities, in order to advance specific understanding of SP effects, there is a need for more sophisticated research that can tease apart the specific missing components from the early environment in case of premature birth while maintaining the rest of the infant's birth conditions the same, similar to the empirical program of Hofer and colleagues. Our longitudinal Kangaroo Care study provides such an example. Guided by the findings that animals deprived of maternal proximity show lifelong deficits in specific systems, including autonomic maturation, the biological clock, HPA response, and PFC-mediated executive control, we randomly selected 73 premature mother-preterm dyads to undergo Kangaroo Care (KC) intervention, providing mother-infant skin-to-skin contact for an hour per day for 14 consecutive days during the period of full incubation. Infants receiving $\mathrm{KC}$ were matched for medical and demographic conditions with 73 mother-preterm dyads receiving standard incubator care. Dyads were seen eight times: prior to $\mathrm{KC}$ and seven times from discharge to 10 years.

The provision of maternal contact during the SP was found to exert long-term effects, specifically on systems open to influences by maternal proximity in mammals. Furthermore, we found that alteration of the mutually influencing field of infant on context during the SP in and of itself initiated a more optimal trajectory that was self-reinforcing over time. Early effects of maternal contact were seen as early as during hospitalization. Following KC, mothers provided more breast milk, which led to greater OT production in mother and child and better infant neurobehavioral maturation and mother-infant interactions at term age (Feldman \& Eidelman, 2003a). Similarly, while infant RSA was comparable between groups prior to $\mathrm{KC}$, maternal contact accelerated maturation of the autonomic nervous system, and infants who received contact had higher RSA, indicating a more mature system that enables a better foundation for the development of regulatory capacities (Feldman \& Eidelman, 2003b).

Across infancy, early maternal contact improved important components of the infant's skills and alongside enhanced critical aspects of parental care in age-appropriate ways. Mother-child synchrony, reciprocity, and attunement were higher at each observation from birth to 2 years, and mothers reported lower depressive symptoms (Feldman, Eidelman, Sirota, \& Weller, 2002). The father-child relationship was also improved, even though fathers did not provide KC, possibly because infants were more regulated and socially engaged (Feldman, Weller, Sirota, \& Eidelman, 2003). Infants' regulatory abilities improved; they were better able to tolerate stress, modulate moments of high arousal, engage in sustained exploration, manage frustration inherent in learning situations, and exercise delayed attention (Feldman, Weller, Sirota, \& Eidelman, 2002). Similarly, across infancy, infants' mental, but not motor, development was improved. Extensive follow-up of behavioral and physiological systems were conducted at 10 years. We found that starting at 5 years, no differences in global IQ were observed between groups; however, children who received $\mathrm{KC}$ as neonates showed better executive abilities at both 5 and 10 years, implying better capacity for cognitive control, flexibility, and mental shifting and greater PFC maturation. These children also showed attenuated stress response at 10 years as indexed by higher RSA at baseline, lower RSA response to stress, and attenuated cortisol response to the Trier Social Stress Test for Children (Feldman, Rosenthal, et al., 2014). Ten-year-old children who received $\mathrm{KC}$ as neonates showed better sleep, as measured by acrigraphs across five consecutive nights, and mother-child interactions during conflict and positive discussion sessions were more synchronous and reciprocal. These findings demonstrate SP effects in humans; providing maternal proximity during specific time windows for its effects improved maturation of these systems. Our findings highlight three principles for intervention informed by human SP research: (a) targeting systems that are individually stable so that improvement in early functioning can impact later functioning via the system's natural stability, (b) providing intervention during SP for the maturation of specific skills, and (c) structuring intervention in ways that provide the missing component from the infant's environment based on SP conceptualization.

Findings related to OT and cortisol. In our cohorts, cortisol was not compared between preterm and full-term infants, but prior research points to disturbances in HPA function in children born preterm (Grunau et al., 2007). Cortisol was measured between preterm infants receiving $\mathrm{KC}$ and controls. No differences emerged in baseline levels, but the stress response of children to the Trier Social Stress Test was attenuated in the $\mathrm{KC}$ group and recovery to baseline was faster (Feldman, Rosenthal, et al., 2014). Salivary OT levels in infants receiving $\mathrm{KC}$ were higher after social contact with their mothers and were longitudinally predicted by the amounts of breast milk children received as neonates, measured in milli- 
liters from hospital charts throughout the hospitalization period. Similarly, the amount of maternal behavior and mother-infant and father-infant synchrony at birth, 3 months, and 6 months predicted OT levels, highlighting the effects of maternal critical provisions during SP, including breast milk and synchrony, for the later functionality of the OT system.

\section{Longitudinal Cohort II: Multiple birth-Triplets, twins, and singletons}

While multifetal gestation is often confounded with premature birth, it is also a distinct and separate risk condition, associated with its unique "missing element" during the SP. Similar to the KC study, our longitudinal follow-up of triplets tested this unique ecology for its provisions and omissions in a case-matched design comparing triplets to matched twins and singletons.

We followed 23 sets of triplets, 23 sets of twins, and 23 singleton infants $(N=138)$ carefully matched for demographic and medical conditions from birth to 10 years. Triplets constitute a unique ecology from an evolutionary perspective, human ecology resulting from assisted reproduction, and nature has not prepared for a setting where the number of infants outnumbers that of parents. Such ecology includes closer physical contact between siblings, even to the extent of constructing a private language (Thorpe, 2006). Animal studies underscored the sociobiological effects of littermates on the pup's physiology (Laviola \& Terranova, 1998) so that full-blown "deprivation" includes separation from both dam and littermates. At the same time, triplets are deprived of a very important component of human parenting, also found in herding animals like sheep: the exclusive bond between mother and child. Synchrony is built on two components: (a) the parent's provision of adequate amounts of parenting behavior and (b) the matching of such behavior to the child's unique patterns and rhythms, which is built on exclusivity. The triplet ecology represents a setting where the amount of parenting behavior is adequate but its synchrony with the signals of each child is low. This stems from the immense burden on parents who need to learn the specific cues of three nonverbal infants while providing for the physical needs of three infants simultaneously.

Consistent with these predictions, at 3 months of age, we found lower levels of synchrony with both mother and father but similar amounts of parental behavior in the triplet group, in the domains of social gaze, affectionate touch, "motherese" high-pitched vocalizations, positive affect, and physical proximity during interactions between parents and each triplet alone. A later marker of the lack of exclusivity was observed during a separation-reunion episode at 1 year. Triplets expressed much less distress to maternal separation and fewer reengagement attempts upon her return, pointing to an altered pattern of attachment. At 2 years, triplets exhibited more behavior problems than did twins and singletons, which were predicted by lower synchrony and separation-reunion behavior (Feldman \& Eidelman, 2004). Other difficulties in the par- enting ecology were also noted across infancy. Parents of triplets reported significantly higher parenting stress across the first year, and in-depth interview at 1 year revealed that mothers, although showing typical involvement in children's well-being, were less familiar with each child's needs and patterns (feeding patterns and sleep rituals). Similarly, repeated observations from birth to 1 year showed lower maternal sensitivity, which predicted lower symbolic competence at 1 year (Feldman, Eidelman, \& Rotenberg, 2004). In cognitive development, we found lower mental development scores (Mental Development Index) at 6, 12, and 24 months compared to twins and singletons (Feldman \& Eidelman, 2005). Overall, the unique triplet ecology across the infancy period underscores the critical importance of exclusivity to infant development.

The triplet condition, however, also highlights another important aspect of human SP research: the potential for reparation during subsequent SP. In particular, the triplet ecology underscores the next SP in social development, the transition from infancy to childhood at the 2- to 5-year stage. At this stage, children typically enter the social world of nonkin adults and peers, and across cultures, children of this age attend some setting that enables peer interactions (Feldman \& Masalha, 2010). This transition provides opportunities to benefit from other benevolent adults and engage in mutual give-and-receive social exchanges with agemates. The development of peer relationships, while drawing on internalization of early parental synchrony and OT, provides a unique context for the activation of the OT system that supports network reorganization via feedforward mechanisms (Feldman, Gordon, et al., 2013). This transition was forecasted in Harlow and Suomi's (1971) seminal work on "peers as therapist" at the transition from infancy to childhood. We found that during the 2-5 transition, triplets showed a catch-up in every developmental domain, including cognitive, social, emotionregulatory, and mental health. This may be because the early rearing context was not inherently pathological, children received adequate amounts of parenting behavior, and the setting was not colored by marked parental psychopathology but by high stress. During the next SP, when other social agents become critical, there seem to be alternative pathways to socialization. At 5 years, no developmental lag was found in cognitive abilities, behavior problems, parental sensitivity, synchrony, social engagement, empathic behavior, or adaptation to childcare, and this catch-up was retained by 10 years (Feldman \& Eidelman, 2009b).

Some triplets, however, did not show the developmental catch-up from 2 to 5 years, and this cohort can thus provide insights on both the capacity for reparation in some cases and the inability of later provisions to alter early delay in others. In approximately two-thirds of triplet sets, one infant suffered intrauterine growth retardation (IUGR) and was smaller than the siblings. This child did not show improved development during the 2-5 transition, and at 5 years still lagged behind on cognitive and social milestones and exhibited more behavior problems. Looking back at the provisions these chil- 
dren received from their parents during the early SP, we found that IUGR children, who are less biologically regulated and require more parental investment to reach typical milestones, received significantly less parental attunement than their siblings. Parents showed lower sensitivity, less parenting behavior, and diminished synchrony toward the IUGR infant as compared to the two siblings (Feldman \& Eidelman, 2005, 2009b). These findings provide an additional angle to the SP conceptualization. It appears that factors related to the inner maturation of the system, genetic makeup, or stage in brain development determine not only the complex relations between infant and context but also the extent to which the sequence of $\mathrm{CP}$ described in molecular research may materialize for each individual child. Because some children are less able than others to utilize the opportunities available in their ecology for developmental catch-up, intervention effort should be directed at better detecting those infants most at risk already at the neonatal stage. There is a need to direct attention to infants who are born prematurely, are part of a multiple gestation, and whose birth weight is $15 \%$ less than their sibling(s) already at birth. With the improvement in assisted reproduction, the increase in maternal age, and the ability of modern medicine to save infants born at increasingly smaller birth weights, the number of such infants is on the rise (triplets are the fastest growing birth population). These findings also provide an empirical example for the aforementioned conceptualization on human-specific SP research. Because the human ecology is immensely variable, research that can compare the growth trajectories of three infants who shared the same gestational environment and are growing up at the same time in the same environment but are still presenting different developmental profiles can provide a unique viewpoint on how environmental effects vary according to the skills each child possesses in eliciting provisions for social-emotional growth.

Findings related to OT and cortisol. The cortisol response of triplets did not differ from that of singletons and twins matched for prematurity. Thus, this condition in and of itself is not shown to be sufficiently stressful, and it can therefore be concluded that it approximates the "peer rearing" ecology described by Harlow and Suomi (1971) only partially. Triplet infants still receive parental investment in healthy families, although the exclusive element is reduced during infancy. We did not measure OT in this sample, so the link between such rearing and OT is not available.

\section{Longitudinal Cohort III: Maternal postpartum depression}

Maternal postpartum depression (PPD) is a condition where the typical increase in maternal depressive symptoms and "blues" following birth found in $85 \%$ of women after childbirth becomes sufficiently severe and lasting to merit Axis I diagnosis of unipolar depression. This condition occurs in approximately $15 \%$ of women in industrial societies, and the numbers appear to increase each decade (Noble, 2005). It has been repeatedly shown that PPD bears long-term consequences for child development, including increased propensity for psychopathology (Goodman \& Gotlib, 1999; Goodman et al., 2011), disruption to the stress response (Ashman, Dawson, Panagiotides, Yamada, \& Wilkinson, 2002), and social difficulties (Carter, Garrity-Rokous, Chazan-Cohen, Little, \& Briggs-Gowan, 2001; Murray et al., 1999). Less is known about the precise mechanisms of cross-generation transmission of psychiatric vulnerability from depressed mothers to their infants because very few studies have utilized longitudinal designs that have included multiple observations of social interactions from birth. Similarly, no study, to our knowledge, has included observations of fathering in the context of maternal depression, and few studies have followed the long-term consequences of specific disruptions in neurobiological systems, such as OT or the stress response. Most important, longitudinal studies on postpartum depression have not teased apart maternal depression from other frequent comorbid risk conditions, such as poverty, single parenthood, substance and alcohol abuse, or premature birth.

Our decade-long follow-up of maternal postpartum depression attempted to follow the research program of Meaney and colleagues (Cameron et al., 2008; Meaney, 2001; Weaver et al., 2004) on high versus low maternal licking and grooming by hypothesizing that the missing environmental component underpinning the long-term effects of maternal depression on child brain and behavior is the dramatic reduction in maternal behavior during the early SP. We attempted to follow Meaney's (2010) methodology, not only the findings, but also the constructed extreme groups built on natural variations in the general population, particularly because PPD is common in the population. We recruited a large cohort $(N$ $=1,983$ ) of women giving birth in maternity wards who completed self-report measures of depression on the first postpartum day. To control for common comorbidities, only healthy women, married or cohabitating, above 21 years, with at least 12 years of education, and above poverty level who gave birth to a term, singleton, and healthy baby were recruited. Using an extreme-case design, women at the high and low ends of the depression continuum were repeatedly tested across the first 9 months following birth and were selected to continue the study, excluding those above cutoff in anxiety levels. This resulted in two matched cohorts of clinically depressed and nondepressed mothers and their children, who were visited at home for a psychiatric diagnosis confirming depression at 9 months, 6 years, and 10 years. During home visits, we conducted clinical evaluation of mother and child, social interactions, emotion regulation paradigms, and genetic and hormonal assessment, and we developed a special battery aimed to assess child social skills, particularly empathy and social engagement. The 6-year visit also included fatherchild interactions, family interactions, and father genes and hormones. We are currently imaging the brains of children of depressed and nondepressed mothers at 11-12 years.

At birth, women who reported high depressive symptoms perceived their infants, the pregnancy, and the maternal role 
less favorably, setting the stage for less involved parenting (Weisman et al., 2010). During home observations at 9 months, depressed mothers showed lower sensitivity, their infants demonstrated less positive affect, and children of depressed mothers were less able to regulate negative emotions (Feldman et al., 2009). Interactions between depressed mothers and their infants were less synchronous, expressed in both lower levels of gaze synchrony and touch synchrony, the coordination of affectionate touch with mutual gazing, and diminished maternal behavior. The latency to the first episode of gaze synchrony, the joint event that signals the start of mutual communication, was five times longer between depressed mothers and their infants, taking $60 \mathrm{~s}$ to onset as compared to $12 \mathrm{~s}$ in controls. We found that mothers tended to break synchronous moments before the infant did, and $75 \%$ of the time, the mother was the first to break mutual gaze. The low synchrony led to disrupted infant emotion regulation in settings that elicited both negative and positive emotions and to lower child positive expression during the emotion-regulation paradigm with the mother and a stranger (Granat et al., 2015).

Both depressed mothers and their infants showed higher baseline cortisol and steeper cortisol response to stress, and less efficient cortisol recovery and biological synchrony was found between maternal and child cortisol (Feldman et al., 2009) at 9 months. Thus already at this age, PPD negatively impacts the infant's stress response through mechanisms of biobehavioral synchrony.

At 6 years, two groups of children were visited $(N=156)$ : those with chronically depressed mothers across the first 6 years $(N=46)$ and those of nondepressed mothers. This enabled us to examine children who received low licking and grooming across the first 6 years of life. Children of chronically depressed mothers had a fourfold increase in the propensity to develop a psychiatric disorder upon entering school. In $60 \%$ of the children to chronically depressed mothers, we found an Axis I psychiatric disorder by school entry, mainly anxiety and conduct disorders, compared to $15 \%$ in children of controls. Children of depressed mothers also exhibited greater social withdrawal and lower empathy and social engagement, as measured by behavioral paradigms (ApterLevy, Feldman, Vakart, Ebstein, \& Feldman, 2013). Those outcomes were moderated by the effects of maternal depression on the child's OT and HPA systems, and the cross-generation transmission of psychiatric vulnerability involved genes, hormones, and diminished maternal synchrony. The high-risk GG allele on OXTR (rs2254298), previously linked with greater risk for autism and depression (Lerer et al., 2008), was overrepresented in depressed mothers, their husbands, and their children, highlighting the genetic susceptibility to depression in this sample. Lower functionality of the OT system was also observed in lower levels of salivary OT in all family members of depressed mothers, including mothers, fathers, and children, and decreased child OT also predicted lower empathy and social engagement in the children. Of interest here, the existence of an A allele on OXTR rs2254298
(AA or AG) in depressed mothers functioned as a resilience factor. Among depressed mothers who were A carriers, there was a 50\% decrease in the propensity for child psychopathology as compared to depressed mothers who were homozygous for the $\mathrm{G}$ allele. This is consistent with the notion that the A allele on this SNP is more evolutionarily recent, which provides a resilience buffer in contexts of early adversity (Brüne, 2012). One possible reason is the findings that A carriers provided more touch to their children during social interactions than those who were $\mathrm{G}$ homozygous. In assessing urinary OT in these mothers and children before and after interactions, we found attenuated OT response in children of depressed mothers, indicating that social contact with depressed mother does not elicit OT response. Similar to the findings for $O X T R$, maternal baseline OT moderated the effect of depression on the child's OT response. Slope analysis indicated that in the context of medium and low maternal baseline OT, child OT response was negatively impacted by maternal depression. However, when mothers had high baseline OT, PPD had no effect on the child's OT, and OT response was high in children of both depressed and nondepressed mothers. Child OT response was predicted by low maternal negative mood and expressed anger. These findings similarly indicate that maternal OT functionality may buffer against the impact of depression on the child's OT system and, consequently, on social growth (Pratt et al., 2014).

In addition to disruptions to the OT system, children of depressed mothers showed aberrant stress response at 6 years. Maternal and child diurnal cortisol and mothers' diurnal DHEA were measured over several days, and maternal and child cortisol response to stress were assessed during the home visit. Children of depressed mothers showed higher diurnal cortisol production and flatter diurnal cortisol variability, and their mothers displayed lower DHEA and a lower DHEA to cortisol ratio. In addition to diurnal patterns, stress reactivity also revealed an effect of maternal depression, and children with psychopathology born to depressed mothers showed an increase, not a decrease, in cortisol at the recovery assessment following stress (Apter-Levi et al., 2014). These findings highlight the effect of chronic maternal depression on both the OT and the HPA systems and are consistent with research in rodents demonstrating alterations to glucocorticoid receptors in the hippocampus (Liu et al., 1997) and low OT receptor distribution in the NAcc (Cameron et al., 2008) among infants of low licking and grooming dams. The findings also demonstrate that interaction with the mother, which typically increases OT and reduces cortisol, does not function in a similar manner for children of depressed mothers. OT levels after social contact with the depressed mother did not increase, and maternal presence failed to buffer the child's stress response. It is hypothesized that this biobehavioral effect to social contact is among the mechanisms that underpin the cross-generation transmission of psychiatric vulnerability from depressed mothers to their children.

Fathering, however, had a protective effect in the context of chronic maternal depression. When mothers were depressed, 
father-child synchrony uniquely predicted lower externalizing and internalizing symptoms, whereas among controls, father's synchrony did not add unique variance beyond maternal-child synchrony. Furthermore, father effects were moderated by the child's genetic risk on the DRD4 gene, indicating that the child's genetic makeup may determine the extent to which fathering can buffer the effects of maternal depression.

Finally, results at 10 years showed that, in the main, maternal depression across early childhood no longer had direct effects on child outcomes. Children's Axis I disorder at 10 years was continuous with psychopathology at 6 years, and the impact on child OT and cortisol response, emotion recognition and regulation, executive functions, and social behavior mainly resulted from the interaction of maternal depression and child psychopathology. These findings have important implications for understanding SP cascades, paralleling the sequence of $\mathrm{CP}$ described in molecular research from global to specific and from lower to higher brain functions (Toyoizumi et al., 2013). Maternal depression in infancy exerts a global negative effect on neurobiological and behavioral outcomes in infancy. During the next SP at 2-5 years, effects become modulated by other attachments (fathers and siblings) and biological aspects of mother and child (e.g., maternal baseline OT, maternal touch, and maternal A allele on OXTR (rs2254298), leading to the consolidation of psychopathology in some children but not in others. In those vulnerable children, more so than in others, psychopathology is likely to self-perpetuate so that at the next transition (6-10 years) psychopathology persists in those who entered the transition with fewer resources. The brain imaging study under way may shed light on networks underpinning social brain development and connectivity in children of depressed mothers who did or did not develop psychopathology to further understand SP cascades. Finally, longitudinal follow-up is required to test how these children meet new transitions, including pubertal transition, leaving home, forming relationships with partners, and eventually nurturing the next generation.

\section{Longitudinal Cohort IV: Repeated unpredictable trauma}

Our decade-long study of children exposed to continuous war-related trauma was set to test the long-term effects of highly unpredictable, potentially lethal circumstances on development, using a birth cohort of children growing up in a specific war zone as a "natural experiment." While it is impossible to compare the degree, intensity, and frequency of childhood trauma across participants, this cohort of children growing up in the same town in Israel (Sderot) bordering the Gaza Strip and exposed to repeated missile attacks can provide some insights on one of the central questions in posttraumatic stress disorder (PTSD) research: what differentiates children exposed to trauma who develop psychopathology, particularly PTSD, from those exposed to the same traumatic experiences who are more resilient.

We recruited 232 families of infants: 156 reared in zones of continuous war and 86 matched controls. Children were seen in infancy, during early childhood, at 7-8 years, and at 10 years, and are currently undergoing brain imaging. Consistent with research on childhood PTSD, we found that maternal psychopathology, including symptoms of anxiety, depression, and PTSD, and maternal support networks play an important role in child psychopathology. Mother's capacity to provide sensitive containment to the child's fears, as observed during both free play and moments of reenactment of the traumatic event, differentiated children who did and did not develop PTSD in early childhood (Feldman \& Vengrober, 2011). Two stress biomarkers were assessed in mothers and children at baseline, following induced stressor, and at recovery: cortisol and sAA, which index the two arms of the stress response, the HPA and sympathetic-adrenomedullary systems (Nater et al., 2005). While war exposure altered the child's stress physiology, patterns of cortisol and sAA differed according to PTSD status. Similar to children in severe adversity (Tarullo \& Gunnar, 2006) and findings on hypocortisolism following extreme trauma (Gunnar \& Vazquez, 2001), children with PTSD exhibited low and flat cortisol levels without the typical reactivity and recovery. Similar low and flat response emerged for sAA, indicating that the two stress-related systems did not work in coordination for a smooth stress response (Allwood, Handwerger, Kivlighan, Granger, \& Stroud, 2011). Exposed children who did not develop PTSD also had a nonreactive stress system, but these exhibited very high, albeit nonresponsive cortisol and sAA, suggesting that chronic early stress reduces variability of the stress response and its reactivity to momentary stressors. Differences between exposed children with and without PTSD were predicted by attachment behavior. While both groups showed higher negative emotionality in a fear paradigm, children with PTSD utilized withdrawal as a mode of emotion regulation, whereas exposed children who were more resilient utilized proximity-seeking behavior to regulate fear (Feldman, Vengrober, et al., 2013).

Findings for the OT system showed that allelic variations on the OT-AVP pathway served as a buffer against stress. In middle childhood, we assessed three risk alleles in mothers, fathers, and children on the OXTR (rs53576, rs2254298, and rs1042778), $C D 38$, an ectoenzyme that mediates the release of OT from hypothalamic neurons through the mobilization of calcium, and AVP receptor 1A (AVPR1A) genes, all previously associated with risk for social dysfunction and less optimal parenting (Feldman et al., 2012; Meyer-Lindenberg et al., 2009), and we computed a cumulative genetic risk index for each family member on the OT-AVP neuropathway. Mother-child sensitivity was observed during trauma evocation and synchrony during free play. Overall, growing up in a zone of continuous war increased child propensity to develop an Axis I psychiatric disorder by middle childhood (Feldman, Vengrober, et al., 2014). Consistent with animal studies on maternal rotation or variable foraging demands, we found a threefold increase in child psychopathology in the war-exposed group. Better functionality on the OT-AVP neuropathway attenuated children's avoidance symptomatology, a central predictor of PTSD chronicity, and the interaction of a 
more functional OT-AVP system and greater maternal sensitivity buffered against the development of psychopathology. Furthermore, maternal, paternal, and child risk on the OTAVP neuropathway differentiated children with chronic PTSD from those whose PTSD in early childhood has remitted by middle childhood.

Follow-up assessment at 10 years continues to show disruptions to the stress response seen in hair cortisol. Assessment of salivary OT show decreased OT production in war-exposed children with internalizing psychopathology and little OT response to social contact. These findings support those from the previous cohort by showing that at this SP, psychopathology is self-perpetuating. Most alarming is the findings that children with chronic PTSD from early to middle childhood, from the 2- to 5-year and 7- to 8-year periods, are much less competent in meeting the next transition, and those show the worst outcomes in terms of the stress and affiliation hormones, relationship with parents, and psychopathology. Moreover, in $66 \%$ of the children diagnosed with PTSD, we found other comorbid disorders (generalized anxiety disorder or conduct disorder), pinpointing those children at highest risk. The brain study currently under way in this cohort may similarly indicate specific networks that render some children exposed to continuous stressors more vulnerable than others.

Overall, results from these four high-risk cohorts provide a unique opportunity to address the complex topic of SP in social development from a multidimensional biobehavioral human perspective. Our findings corroborate animal research on the critical importance of the early environment, on the role of maternal proximity in shaping the OT and HPA systems, and on the cascade of SPs from global to local and from lower to higher functioning. However, the findings also accord with principles of developmental psychopathology (Cicchetti \& Toth, 2009) and depict an infinitely complex human-specific picture, with later SP enabling reparation to some children but not to others. Our findings also highlight the feedforward mechanisms by which children can utilize later relationships for social growth, and the tendency of psychopathology to become entrenched over time, constrain the system's future options, and lead to reduced plasticity. These unique cohorts running in parallel in the same lab and utilizing the same procedures also provide an opportunity to look back into the rich data collected to detect seemingly minor variations in the early environment that can account for disorders that remit, disorders that are chronic, and resilience following significant disruptions to the early environment during SP for human social growth.

\section{Conclusions}

This paper proposes a rigorous program for human SP research based on the multifinality and infinite possibilities embedded in the human brain. While much research on SP, risk, and resilience is rooted in evolutionary thinking, Hofer (2006) reminds us that evolutionary perspective on human development must be complemented with insights and from other domains, such as the humanities, social sciences, and clinical wisdom (Cicchetti \& Toth, 2009). While evolution is impartial to the individual child, keeping sight of the individual is precisely what matters for developmental science. The goal of a human program set to understand how early environments meet or fail the needs of their infants is to enable the individual to benefit from the fullness of human experience afforded by modern science: long time to maturity, planned parenthood, freedom from infectious disease, literacy, and manageable stress response. Human research must translate into human intervention, document the missing components across variable early environments, and attempt to disseminate knowledge and develop interventions that are clinically efficient yet maintain deepest respect for the individual's local ecology, cultural heritage, social meaning system, and personal life history.

\section{References}

Abraham, E., Hendler, T., Shapira-Lichter, I., Kanat-Maymon, Y., ZagoorySharon, O., \& Feldman, R. (2014). Father's brain is sensitive to childcare experiences. Proceedings of the National Academy of Sciences, 111, 9792-9797.

Adkins-Regan, E. (1998). Hormonal mechanisms of mate choice. Integrative and Comparative Biology, 38, 166-178.

Allison, T., Puce, A., \& McCarthy, G. (2000). Social perception from visualcues: Role of the STS region. Trends in Cognitive Sciences, 4, 267-278.

Allwood, M., Handwerger, K., Kivlighan, K. T., Granger, D. A., \& Stroud, L. R. (2011). Direct and moderating links of salivary alpha-amylase and cortisol stress-reactivity to youth behavioral and emotional adjustment. Biological Psychology, 88, 57-64.

Apter-Levi, Y., Zagoory-Sharon, O., \& Feldman, R. (2014). Oxytocin and vasopressin support distinct configurations of social synchrony. Brain Research, 1580, 124-132.

Apter-Levy, Y., Feldman, M., Vakart, A., Ebstein, R. P., \& Feldman, R. (2013). Impact of maternal depression across the first 6 years of life on the child's mental health, social engagement, and empathy: The moderating role of oxytocin. American Journal of Psychiatry, 170, 1161-1168.

Ashman, S. B., Dawson, G., Panagiotides, H., Yamada, E., \& Wilkinson, C. W. (2002). Stress hormone levels of children of depressed mothers. Development and Psychopathology, 14, 333-349.
Atzil, S., Hendler, T., \& Feldman, R. (2011). Specifying the neurobiological basis of human attachment: Brain, hormones and behavior in synchronous and intrusive mothers. Neuropsychopharmacology, 36, 26032615.

Atzil, S., Hendler, T., \& Feldman, R. (2014). The brain basis of social synchrony. Social Cognitive and Affective Neuroscience, 9, 1193-1202.

Atzil, S., Hendler, T., Zagoory-Sharon, O., Winetraub, Y., \& Feldman, R. (2012). Synchrony and specificity in the maternal and the paternal brain: Relations to oxytocin and vasopressin. Journal of the American Academy of Child \& Adolescent Psychiatry, 51, 798-811.

Bakermans-Kranenburg, M. J., \& van IJzendoorn, M. H. (2011). Differential susceptibility to rearing environment depending on dopamine-related genes: New evidence and a meta-analysis. Development and Psychopathology, 23, 39-52.

Bales, K. L., \& Carter, C. S. (2003). Developmental exposure to oxytocin facilitates partner preferences in male prairie voles. Behavioral Neuroscience, 117, 854-859.

Bali, B., \& Kovacs, K. J. (2003). GABAergic control of neuropeptide gene expression in parvocellular neurons of the hypothalamic paraventricular nucleus. European Journal of Neuroscience, 18, 1518-1526.

Bartal, I. B.-A., Decety, J., \& Mason, P. (2011). Helping a cagemate in need: Empathy and pro-social behavior in rats. Science, 334, 1427-1430. 
Beets, I., Temmerman, L., Janssen, T., \& Schoofs, L. (2013). Ancient neuromodulation by vasopressin/oxytocin-related peptides. Worm, 2, e24246.

Bernhardt, B. C., \& Singer, T. (2012). The neural basis of empathy. Annual Review of Neuroscience, 35, 1-23.

Bloom, H. (1980). Wallace Stevens: The poems of our climate. Ithaca, NY: Cornell University Press.

Blyth, B. J., Hauger, R. L., Purdy, R. H., \& Amico, J. A. (2000). The neurosteroid allopregnanolone modulates oxytocin expression in the hypothalamic paraventricular nucleus. American Journal of Physiology: Regulatory, Integrative and Comparative Physiology, 278, R684R691.

Bosch, O. J., Meddle, S. L., Beiderbeck, D. I., Douglas, A. J., \& Neumann, I. D. (2005). Brain oxytocin correlates with maternal aggression: Link to anxiety. Journal of Neuroscience, 25, 6807-6815.

Bosch, O. J., Waldherr, M., Nair, H. P., Young, L. J., \& Neumann, I. D. (2006). Viral vector-mediated overexpression of oxytocin receptors in the amygdala of virgin rats increases aggression and reduces anxiety. Frontiers in Neuroendocrinology, 27, 124-125.

Bowlby, J. (1953). Some pathological processes set in train by early motherchild separation. Journal of Mental Science, 2, 265-272.

Bowlby, J. (1958). The nature of the child's tie to his mother. International Journal of Psychonalysis, 39, 350-373.

Brazelton, T. B., Koslowski, B., \& Main, M. (1974). The origins of reciprocity: The early mother-infant interaction. New York: Wiley-Interscience.

Brüne, M. (2012). Does the oxytocin receptor (OXTR) polymorphism (rs2254298) confer "vulnerability" for psychopathology or "differential susceptibility"? Insights from evolution. BMC Medicine, 10, 38.

Brunton, P. J., \& Russell, J. A. (2008). The expectant brain: Adapting for motherhood. Nature Neuroscience, 9, 11-25.

Burgdorf, J., \& Panksepp, J. (2006). The neurobiology of positive emotions. Neuroscience \& Biobehavioral Reviews, 30, 173-187.

Cameron, N. M., Shahrokh, D., Del Corpo, A., Dhir, S. K., Szyf, M., Champagne, F. A. et al. (2008). Epigenetic programming of phenotypic variations in reproductive strategies in the rat through maternal care. Journal of Neuroendocrinology, 20, 795-801.

Camus, A. (1994). The first man. Harmondsworth: Penguin.

Carter, A. S., Garrity-Rokous, F. E., Chazan-Cohen, R., Little, C., \& BriggsGowan, M. J. (2001). Maternal depression and comorbidity: Predicting early parenting, attachment security, and toddler social-emotional problems and competencies. Journal of the American Academy of Child \& Adolescent Psychiatry, 40, 18-26.

Carter, C. S. (1998). Neuroendocrine perspectives on social attachment and love. Psychoneuroendocrinology, 23, 779-818.

Carter, C. S. (2014). Oxytocin pathways and the evolution of human behavior. Annual Review of Psychology, 65, 17-39.

Carter, C. S., Altemus, M., \& Chrousos, G. P. (2001). Neuroendocrine and emotional changes in the post-partum period. Progress in Brain Research, 133, 241-249.

Carter, C. S., \& Porges, S. W. (2013). The biochemistry of love: An oxytocin hypothesis. EMBO Reports, 14, 12-16.

Champagne, F. A., Weaver, I. C. G., Diorio, J., Dymov, S., Szyf, M., \& Meaney, M. J. (2006). Maternal care associated with methylation of the estrogen receptor-alphalb promoter and estrogen receptor-alpha expression in the medial preoptic area of female offspring. Endocrinology, 147, 2909-2915.

Cicchetti, D. (2013). Annual Research Review: Resilient functioning in maltreated children-Past, present, and future perspectives. Journal of Child Psychology and Psychiatry and Allied Disciplines, 54, 402-422.

Cicchetti, D., \& Toth, S. L. (2009). The past achievements and future promises of developmental psychopathology: The coming of age of a discipline. Journal of Child Psychology and Psychiatry and Allied Disciplines, 50, 16-25.

Cicchetti, D., \& Tucker, D. (1994). Development and self-regulatory structures of the mind. Development and Psychopathology, 6, 533-549.

Clodi, M., Vila, G., Riedl, M., Stulnig, T. M., Struck, J., Luger, T. A. et al. (2008). Oxytocin alleviates the neuroendocrine and cytokine response to bacterial endotoxin in healthy men. American Journal of Endocrinology and Metabolism, 295, 686-691.

Coplan, J. D., Andrews, M. W., Rosenblum, L. A., Owens, M. J., Friedman, S., Gorman, J. M. et al. (1996). Persistent elevations of cerebrospinal fluid concentrations of corticotropin-releasing factor in adult nonhuman primates exposed to early-life stressors: Implications for the pathophysiology of mood and anxiety disorders. Proceedings of the National Academy of Sciences, 9, 1619-1623.
Cushing, B. S., \& Carter, C. S. (2000). Peripheral pulses of oxytocin increase partner preferences in female, but not male, prairie voles. Hormones and Behavior, 37, 49-56.

Dabrowska, J., Hazra, R., Ahern, T. H., Guo, J.-D., McDonald, A. J., Mascagni, F. et al. (2011). Neuroanatomical evidence for reciprocal regulation of the corticotrophin-releasing factor and oxytocin systems in the hypothalamus and the bed nucleus of the stria terminalis of the rat: Implications for balancing stress and affect. Psychoneuroendocrinology, 36, 1312-1326.

Dahlen, H. G., Kennedy, H. P., Anderson, C. M., Bell, A. F., Clark, A., Foureur, M. et al. (2013). The EPIIC hypothesis: Intrapartum effects on the neonatal epigenome and consequent health outcomes. Medical Hypotheses, 80, 656-662.

Daskalakis, N. P., Bagot, R. C., Parker, K. J., Vinkers, C. H., \& de Kloet, E. R. (2013). The three-hit concept of vulnerability and resilience: Toward understanding adaptation to early-life adversity outcome. Psychoneuroendocrinology, 38, 1858-1873.

de Jong, T. R., Chauke, M., Harris, B. N., \& Saltzman, W. (2009). From here to paternity: Neural correlates of the onset of paternal behavior in California mice (Peromyscus californicus). Hormones and Behavior, 56, 220-231.

Denenberg, V. H., \& Bell, R. W. (1960). Critical periods for the effects of infantile experience on adult learning. Science, 131, 227-228.

Denenberg, V. H., Hudgens, G. A., \& Zarrow, M. (1964). Mice reared with rats: Modification of behavior by early experience with another species. Science, 143, 380-381.

Denenberg, V. H., Ottinger, D. R., \& Stephens, M. W. (1962). Effects of maternal factors upon growth and behavior of the rat. Child Development, $33,65-71$.

Dölen, G., Darvishzadeh, A., Huang, K. W., \& Malenka, R. C. (2013). Social reward requires coordinated activity of nucleus accumbens oxytocin and serotonin. Nature, 501, 179-184.

Donaldson, Z. R., \& Young, L. J. (2008). Oxytocin, vasopressin, and the neurogenetics of sociality. Science, 322, 900-904.

Edelman, G. (2004). Wider than the sky: The phenomenal gift of consciousness. New Haven, CT: Yale University Press.

Elbert, T., Pantev, C., Wienbruch, C., Rockstroh, B., \& Taub, E. (1995). Increased cortical representation of the fingers of the left hand in string players. Science, 270, 305-307.

Erikson, E. H. (1963). Childhood and society. New York: Stonton.

Feldman, R. (2003). Infant-mother and infant-father synchrony: The coregulation of positive arousal. Infant Mental Health Journal, 24, 1-23.

Feldman, R. (2007a). Parent-infant synchrony and the construction of shared timing: Physiological precursors, developmental outcomes, and risk conditions. Journal of Child Psychology and Psychiatry and Allied Disciplines, 48, 329-354.

Feldman, R. (2007b). On the origins of background emotions: From affect synchrony to symbolic expression. Emotion, 7, 601-611.

Feldman, R. (2007c). Mother-infant synchrony and the development of moral orientation in childhood and adolescence: Direct and indirect mechanisms of developmental continuity. American Journal of Orthopsychiatry, 77, 582-597.

Feldman, R. (2009). The development of regulatory functions from birth to 5 years: Insights from premature infants. Child Development, 80, 544-561.

Feldman, R. (2010). The relational basis of adolescent adjustment: Trajectories of mother-child interactive behaviors from infancy to adolescence shape adolescents' adaptation. Attachment and Human Development, 12, 173-192.

Feldman, R. (2012a). Oxytocin and social affiliation in humans. Hormones and Behavior, 61, 380-391.

Feldman, R. (2012b). Bio-behavioral synchrony: A model for integrating biological and microsocial behavioral processes in the study of parenting. Parenting, 12, 154-164.

Feldman, R. (2012c). Parent-infant synchrony: A biobehavioral model of mutual influences in the formation of affiliative bonds. Monographs of the Society for Research in Child Development, 77, 42-51.

Feldman, R. (2013). Synchrony and the neurobiological basis of social affiliation. In M. Mikulincer \& P. R. Shaver (Eds.), Mechanisms of social connection: From brain to group (pp. 145-166). Washington, DC: American Psychological Association.

Feldman, R. (2015). Mutual influences between child emotion regulation and parent-child reciprocity shape development across the first decade of life: Implications for developmental psychopathology. Manuscript submitted for publication.

Feldman, R., Bamberger, E., \& Kanat-Maymon, Y. (2013). Parent-specific reciprocity from infancy to adolescence shapes children's social compe- 
tence and dialogical skills. Attachment and Human Development, 15, 407-423.

Feldman, R., \& Eidelman, A. I. (2003a). Direct and indirect effects of breast milk on the neurobehavioral and cognitive development of premature infants. Developmental Psychobiology, 43, 109-119.

Feldman, R., \& Eidelman, A. I. (2003b). Skin-to-skin contact accelerates autonomic and neurobehavioural maturation in preterm infants. Developmental Medicine and Child Neurology, 45, 274-281.

Feldman, R., \& Eidelman, A. I. (2004). Parent-infant synchrony and the social-emotional development of triplets. Developmental Psychology, 40, 1133-1147.

Feldman, R., \& Eidelman, A. I. (2005). Does a triplet birth pose a special risk for infant development? Assessing cognitive development in relation to intrauterine growth and mother-infant interaction across the first 2 years. Pediatrics, 115, 443-452.

Feldman, R., \& Eidelman, A. I. (2007). Maternal postpartum behavior and the emergence of infant-mother and infant-father synchrony in preterm and full-term infants: The role of neonatal vagal tone. Developmental Psychobiology, 49, 290-302.

Feldman, R., \& Eidelman, A. I. (2009a). Biological and environmental initial conditions shape the trajectories of cognitive and social-emotional development across the first years of life. Developmental Science, 12, 194-200.

Feldman, R., \& Eidelman, A. I. (2009b). Triplets across the first 5 years: The discordant infant at birth remains at developmental risk. Pediatrics, 124, 316-323.

Feldman, R., Eidelman, A. I., \& Rotenberg, N. (2004). Parenting stress, infant emotion regulation, maternal sensitivity, and the cognitive development of triplets: A model for parent and child influences in a unique ecology. Child Development, 75, 1774-1791.

Feldman, R., Eidelman, A. I., Sirota, L., \& Weller, A. (2002). Comparison of skin-to-skin (Kangaroo) and traditional care: Parenting outcomes and preterm infant development. Pediatrics, 110, 16-26.

Feldman, R., Gordon, I., Influs, M., Gutbir, T., \& Ebstein, R. P. (2013). Parental oxytocin and early caregiving jointly shape children's oxytocin response and social reciprocity. Neuropsychopharmacology, 38, 1154-1162.

Feldman, R., Gordon, I., Schneiderman, I., Weisman, O., \& Zagoory-Sharon, O. (2010). Natural variations in maternal and paternal care are associated with systematic changes in oxytocin following parent-infant contact. Psychoneuroendocrinology, 35, 1133-1141.

Feldman, R., Gordon, I., \& Zagoory-Sharon, O. (2010). The cross-generation transmission of oxytocin in humans. Hormones and Behavior, 58, 669-676.

Feldman, R., Granat, A., Pariente, C., Kanety, H., Kuint, J., \& GilboaSchechtman, E. (2009). Maternal depression and anxiety across the postpartum year and infant social engagement, fear regulation, and stress reactivity. Journal of the American Academy of Child \& Adolescent Psychiatry, 48, 919-927.

Feldman, R., Greenbaum, C. W., \& Yirmiya, N. (1999). Mother-infant affect synchrony as an antecend of the emergence of self-control. Developmental Psychology, 35, 223-231.

Feldman, R., Magori-Cohen, R., Galili, G., Singer, M., \& Louzoun, Y. (2011). Mother and infant coordinate heart rhythms through episodes of interaction synchrony. Infant Behavior and Development, 34, 569-577.

Feldman, R., \& Masalha, S. (2010). Parent-child and triadic antecedents of children's social competence: Cultural specificity, shared process. Developmental Psychology, 46, 455-467.

Feldman, R., Rosenthal, Z., \& Eidelman, A. I. (2014). Maternal-preterm skinto-skin contact enhances child physiologic organization and cognitive control across the first 10 years of life. Biological Psychiatry, 75, 56-64.

Feldman, R., Singer, M., \& Zagoory, O. (2010). Touch attenuates infants' physiological reactivity to stress. Developmental Science, 13, 271-278.

Feldman, R., \& Vengrober, A. (2011). Posttraumatic stress disorder in infants and young children exposed to war-related trauma. Journal of the American Academy of Child \& Adolescent Psychiatry, 50, 645-658.

Feldman, R., Vengrober, A., \& Ebstein, R. P. (2014). Affiliation buffers stress: Cumulative genetic risk in oxytocin-vasopressin genes combines with early caregiving to predict PTSD in war-exposed young children. Translational Psychiatry, 4, e370.

Feldman, R., Vengrober, A., Eidelman-Rothman, M., \& Zagoory-Sharon, O. (2013). Stress reactivity in war-exposed young children with and without posttraumatic stress disorder: Relations to maternal stress hormones, parenting, and child emotionality and regulation. Development and Psychopathology, 25, 943-955.

Feldman, R., Weller, A., Sirota, L., \& Eidelman, A. I. (2002). Skin-to-Skin contact (Kangaroo Care) promotes self-regulation in premature infants:
Sleep-wake cyclicity, arousal modulation, and sustained exploration. Developmental Psychology, 38, 194.

Feldman, R., Weller, A., Sirota, L., \& Eidelman, A. I. (2003). Testing a family intervention hypothesis: The contribution of mother-infant skin-toskin contact (Kangaroo Care) to family interaction, proximity, and touch. Journal of Family Psychology, 17, 94.

Feldman, R., Zagoory-Sharon, O., Weisman, O., Schneiderman, I., Gordon, I., Maoz, R. et al. (2012). Sensitive parenting is associated with plasma oxytocin and polymorphisms in the OXTR and CD38 genes. Biological Psychiatry, 72, 175-181.

Ferguson, J. N., Aldag, J. M., Insel, T. R., \& Young, L. J. (2001). Oxytocin in the medial amygdala is essential for social recognition in the mouse. Journal of Neuroscience, 21, 8278-8285.

Fogel, A. (1993). Developing through relationships. Chicago: University of Chicago Press.

Francis, D. D., Young, L. J., Meaney, M. J., \& Insel, T. R. (2002). Naturally occurring differences in maternal care are associated with the expression of oxytocin and vasopressin (V1a) receptors: Gender differences. Journal of Neuroendocrinology, 14, 349-353.

Gee, D. G., Humphreys, K. L., Flannery, J., Goff, B., Telzer, E. H., Shapiro, M. et al. (2013). A developmental shift from positive to negative connectivity in human amygdala-prefrontal circuitry. Journal of Neuroscience, $33,4584-4593$.

Gimpl, G., \& Fahrenholz, F. (2001). The oxytocin receptor system: Structure, function, and regulation. Physiological Reviews, 81, 629-683.

Goodman, S. H., \& Gotlib, I. H. (1999). Risk for psychopathology in the children of depressed mothers: A developmental model for understanding mechanisms of transmission. Psychological Review, 106, 458-490.

Goodman, S. H., Rouse, M. H., Connell, A. M., Broth, M. R., Hall, C. M., \& Heyward, D. (2011). Maternal depression and child psychopathology: A meta-analytic review. Clinical Child and Family Psychology Review, 14, $1-27$.

Goodson, J. L. (2013). Deconstructing sociality, social evolution and relevant nonapeptide functions. Psychoneuroendocrinology, 38, 465-478.

Goodson, J. L., Kelly, A. M., \& Kingsbury, M. A. (2012). Evolving nonapeptide mechanisms of gregariousness and social diversity in birds. Hormones and Behavior, 61, 239-250.

Gordon, I., Pratt, M., Zagoory-Sharon, O., \& Feldman, R. (2015). Mutual influences between oxytocin and testosterone shape the development of human parental care. Manuscript submitted for publication.

Gordon, I., Zagoory-Sharon, O., Leckman, J. F., \& Feldman, R. (2010a). Oxytocin and the development of parenting in humans. Biological Psychiatry, 68, 377-382.

Gordon, I., Zagoory-Sharon, O., Leckman, J. F., \& Feldman, R. (2010b). Oxytocin, cortisol, and triadic family interactions. Physiology \& Behavior, 101, 679-684.

Gottman, J. M. (1998). Psychology and the study of the marital processes. Annual Review of Psychology, 49, 169-197.

Gouin, J.-P., Carter, C. S., Pournajafi-Nazarloo, H., Glaser, R., Malarkey, W. B., Loving, T. J. et al. (2010). Marital behavior, oxytocin, vasopressin, and wound healing. Psychoneuroendocrinology, 35, 1082-1090.

Granat, A., Gadassi, R., Gilboa-Schechtman, E., \& Feldman, R. (2015). Maternal depression and anxiety, social synchrony, and infant regulation of negative and positive emotions. Manuscript submitted for publication.

Grunau, R. E., Haley, D. W., Whitfield, M. F., Weinberg, J., Yu, W., \& Thiessen, P. (2007). Altered basal cortisol levels at 3, 6, 8 and 18 months in infants born at extremely low gestation age. Journal of Pediatrics, 150, $151-156$.

Gunnar, M. R., \& Vazquez, D. M. (2001). Low cortisol and a flattening of expected daytime rhythm: Potential indices of risk in human development. Development and Psychopathology, 13, 515-538.

Gur, R., Tendler, A., \& Wagner, S. (2014). Long-term social recognition memory is mediated by oxytocin-dependent synaptic plasticity in the medial amygdala. Biological Psychiatry. Advance online publication.

Haber, S. N., \& Knutson, B. (2010). The reward circuit: Linking primate anatomy and human imaging. Neuropsychopharmacology, 35, 4-26.

Hammock, E. D. (2014). Developmental perspectives on oxytocin and vasopressin. Neuropsychopharmacology. Advance online publication.

Harlow, H. F. (1958). The nature of love. American Psychologist, 13, 673.

Harlow, H. F., \& Suomi, S. J. (1971). Social recovery by isolation-reared monkeys. Proceedings of the National Academy of Sciences, 68, 15341538

Harrison, N. A., Brydon, L., Walker, C., Gray, M. A., Steptoe, A., \& Critchley, H. D. (2009). Inflammation causes mood changes through alterations 
in subgenual cingulate activity and mesolimbic connectivity. Biological Psychiatry, 66, 407-414.

Hensch, T. K. (2005). Critical period plasticity in local cortical circuits. $\mathrm{Na}$ ture Reviews Neuroscience, 6, 877-888.

Hensch, T. K., Fagiolini, M., Mataga, N., Stryker, M. P., Baekkeskov, S., \& Kash, S. F. (1998). Local GABA circuit control of experience-dependent plasticity in developing visual cortex. Science, 282, 1504-1508.

Higashida, H., Yokoyama, S., Kikuchi, M., \& Munesue, T. (2012). CD38 and its role in oxytocin secretion and social behavior. Hormones and Behavior, 61, 351-358.

Hofer, M. A. (1970a). Environmental influences on the early development of autonomic cardiac regulation in the rat. Psychotherapy and Psychosomatics, 18, 243-247.

Hofer, M. A. (1970b). Physiological responses of infant rats to separation from their mothers. Science, 168, 871-873.

Hofer, M. A. (1987). Early social relationships: A psychobiologist's view. Child Development, 58, 633-647.

Hofer, M. A. (1995). Hidden regulators. Attachment Theory: Social, Developmental and Clinical Perspectives, 59, 203-230.

Hofer, M. A. (2006). Evolutionary basis of adaptation in resilience and vulnerability: Response to Cicchetti and Blender. Annals of the New York Academy of Sciences, 1094, 259-262.

Hofer, M. A., \& Shair, H. N. (1987). Isolation distress in two-week-old rats: Influence of home cage, social companions, and prior experience with littermates. Developmental Psychobiology, 20, 465-476.

Insel, T. R. (2010). The challenge of translation in social neuroscience: A review of oxytocin, vasopressin, and affiliative behavior. Neuron, 65, 768-779.

Insel, T. R., \& Young, L. J. (2001). The neurobiology of attachment. Nature Reviews Neuroscience, 2, 129-136.

Johnston, C. C., Stevens, B., Craig, K. D., \& Grunau, R. V. (1993). Developmental changes in pain expression in premature, full-term, two- and four-month-old infants. Pain, 52, 201-208.

Kendrick, K. M. (2013). Oxytocin regulation of sheep social and maternal behavior. In Oxytocin, vasopressin and related peptides in the regulation of behavior (pp. 183-191). New York: Cambridge University Press.

Keverne, E. B. (2013). Importance of the matriline for genomic imprinting, brain development and behaviour. Philosophical Transactions of the Royal Society of London: Series B, Biological Sciences, 368, 20110327.

Keverne, E. B., \& Kendrick, K. M. (1992). Oxytocin facilitation of maternal behavior in sheep. Annals of the New York Academy of Sciences, 652, 83-101.

Kim, K. H., Relkin, N. R., Lee, K. M., \& Hirsch, J. (1997). Distinct cortical areas associated with native and second languages. Nature, 388, 171-174.

Kim, P., Leckman, J. F., Mayes, L. C., Newman, M.-A., Feldman, R., \& Swain, J. E. (2010). NIH Public Access, 13, 662-673.

Kim, P., Rigo, P., Mayes, L. C., Feldman, R., Leckman, J. F., \& Swain, J. E. (2014). Neural plasticity in fathers of human infants. Social Neuroscience (July), 1-14.

Knudsen, E. I. (2004). Sensitive periods in the development of the brain and behavior. Journal of Cognitive Neuroscience, 16, 1412-1425.

Kojima, S., Stewart, R. A., Demas, G. E., \& Alberts, J. R. (2012). Maternal contact differentially modulates central and peripheral oxytocin in rat pups during a brief regime of mother-pup interaction that induces a filial huddling preference. Journal of Neuroendocrinology, 24, 831-840.

Kozorovitskiy, Y., Hughes, M., Lee, K., \& Gould, E. (2006). Fatherhood affects dendritic spines and vasopressin V1a receptors in the primate prefrontal cortex. Nature Neuroscience, 9, 1094-1095.

Latysheva, N. V, \& Rayevsky, K. S. (2003). Chronic neonatal N-methyl-D-aspartate receptor blockade induces learning deficits and transient hypoactivity in young rats. Progress in Neuro-Psychopharmacology and Biological Psychiatry, 27, 787-794.

Laviola, G., \& Terranova, M. L. (1998). The developmental psychobiology of behavioural plasticity in mice: The role of social experiences in the family unit. Neuroscience \& Biobehavioral Reviews, 23, 197-213.

Lee, P. R., Brady, D. L., Shapiro, R. A., Dorsa, D. M., \& Koenig, J. I. (2007). Prenatal stress generates deficits in rat social behavior: Reversal by oxytocin. Brain Research, 1156, 152-167.

Lerer, E., Levi, S., Salomon, S., Darvasi, A., Yirmiya, N., \& Ebstein, R. P. (2008). Association between the oxytocin receptor (OXTR) gene and autism: Relationship to Vineland Adaptive Behavior Scales and cognition. Molecular Psychiatry, 13, 980-988.

Leuner, B., Caponiti, J. M., \& Gould, E. (2012). Oxytocin stimulates adult neurogenesis even under conditions of stress and elevated glucocorticoids. Hippocampus, 22, 861-868. doi:10.1002/hipo.20947
Levine, S. (1956). A further study of infantile handling and adult avoidance learning. Journal of Personality, 25, 70-80.

Levine, S. (1967). Maternal and environmental influences on the adrenocortical response to stress in weanling rats. Science, 156, 258-260.

Levine, S. (2005). Developmental determinants of sensitivity and resistance to stress. Psychoneuroendocrinology, 30, 939-946.

Levine, S., Alpert, M., \& Lewis, G. W. (1957). Infantile experience and the maturation of the pituitary adrenal axis. Science, 126, 1347.

Lightman, S. L., Windle, R. J., Wood, S. A., Kershaw, Y. M., Shanks, N., \& Ingram, C. D. (2001). Peripartum plasticity within the hypothalamopituitary-adrenal axis. Progress in Brain Research, 133, 111-129.

Liu, D., Diorio, J., Tannenbaum, B., Caldji, C., Francis, D., Freedman, A. et al. (1997). Maternal care, hippocampal glucocorticoid receptors, and hypothalamic-pituitary-adrenal responses to stress. Science, 277, $1659-1662$

Liu, H.-X., Lopatina, O., Higashida, C., Tsuji, T., Kato, I., Takasawa, S. et al. (2008). Locomotor activity, ultrasonic vocalization and oxytocin levels in infant CD38 knockout mice. Neuroscience Letters, 448, 67-70.

Lorenz, K. (1935). Der Kumpan in der Umwelt des Vogels. Journal Für Ornithologie, 83, 137-213.

Lorenz, K. (1950). Comparative method in studying innate behaviour patterns. In Company of Biologists (Ed.), Physiological mechanisms in animal behaviour (Vol. 4). Cambridge: Cambridge University Press.

Love, T. M., Enoch, M.-A., Hodgkinson, C. A., Pecina, M., Mickey, B., Koeppe, R. A. et al. (2012). Oxytocin gene polymorphisms influence human dopaminergic function in a sex-dependent manner. Biological Psychiatry, 72, 198-206.

Lovejoy, A. O. (1936). The great chain of being. Cambridge, MA: Harvard University Press.

Ludwig, M., \& Leng, G. (2006). Dendritic peptide release and peptide-dependent behaviours. Nature Reviews Neuroscience, 7, 126-136.

Maestripieri, D., Hoffman, C. L., Anderson, G. M., Carter, C. S., \& Higley, J. D. (2009). Mother-infant interactions in free-ranging rhesus macaques: Relationships between physiological and behavioral variables. Physiology \& Behavior, 96, 613-619.

Mar, R. A. (2011). The neural bases of social cognition and story comprehension. Annual Review of Psychology, 62, 103-134.

Marchini, G., \& Stock, S. (1996). Pulsatile release of oxytocin in newborn infants. Reproduction, Fertility and Development, 8, 163-165.

Margoliash, D. (2002). Evaluating theories of bird song learning: Implications for future directions. Journal of Comparative Physiology, 188A, 851-866.

Meaney, M. J. (2001). Maternal care, gene expression, and the transmission of individual differences in stress reactivity across generation. Annual Review of Neuroscience, 24, 1162-1192.

Meaney, M. J. (2010). Epigenetics and the biological definition of gene $x$ environment interactions. Child Development, 81, 41-79.

Meyer-Lindenberg, A., Domes, G., Kirsch, P., \& Heinrichs, M. (2011). Oxytocin and vasopressin in the human brain: Social neuropeptides for translational medicine. Nature Reviews Neuroscience, 12, 524-538.

Meyer-Lindenberg, A., Kolachana, B., Gold, B., Olsh, A., Nicodemus, K. K., Mattay, V. et al. (2009). Genetic variants in AVPR1A linked to autism predict amygdala activation and personality traits in healthy humans. Molecular Psychiatry, 14, 968-975.

Murray, L., Sinclair, D., Cooper, P., Ducournau, P., Turner, P., \& Stein, A. (1999). The socioemotional development of 5-year-old children of postnatally depressed mothers. Journal of Child Psychology and Psychiatry and Allied Disciplines, 40, 1259-1271.

Nater, U. M., Rohleder, N., Gaab, J., Berger, S., Jud, A., Kirschbaum, C. et al. (2005). Human salivary alpha-amylase reactivity in a psychosocial stress paradigm. International Journal of Psychophysiology, 55, 333342.

Nelson, E., \& Panksepp, J. (1996). Oxytocin and infant-mother bonding in rats. Behavioral Neuroscience, 110, 583-592.

Neumann, I. D. (2008). Brain oxytocin: A key regulator of emotional and social behaviours in both females and males. Journal of Neuroendocrinology, 20, 858-865.

Noble, R. E. (2005). Depression in women. Metabolism, 54, 49-52.

Noonan, L. R., Caldwell, J. D., Li, L., Walker, C. H., Pedersen, C. A., \& Mason, G. A. (1994). Neonatal stress transiently alters the development of hippocampal oxytocin receptors. Developmental Brain Research, 80, 115-120.

Numan, M. (2006). Hypothalamic neural circuits regulating maternal responsiveness toward infants. Behavioral and Cognitive Neuroscience Review, $5,163-190$. 
Numan, M., Numan, M. J., Schwarz, J. M., Neuner, C. M., Flood, T. F., \& Smith, C. D. (2005). Medial preoptic area interactions with the nucleus accumbens-ventral pallidum circuit and maternal behavior in rats. Behavior \& Brain Research, 158, 53-68.

Owen, S. F., Tuncdemir, S. N., Bader, P. L., Tirko, N. N., Fishell, G., \& Tsien, R. W. (2013). Oxytocin enhances hippocampal spike transmission by modulating fast-spiking interneurons. Nature, 500, 458-462.

Pert, C. B., Ruff, M. R., Weber, R. J., \& Herkenham, M. (1985). Neuropeptides and their receptors: A psychosomatic network. Journal of Immunology, 135(Suppl.), 820s-826s.

Pow, D. V., \& Morris, J. F. (1989). Dendrites of hypothalamic magnocellular neurons release neurohypophysial peptides by exocytosis. Neuroscience, $32,435-439$.

Pratt, M., Apter-Levi, Y., Vakart, A., Feldman, M., Fishman, R., Feldman, T., et al. (2015). The cross-generation transmission of affiliative biology: Effect of maternal depression on child oxytocin response is moderated by maternal oxytocin and relational behavior. Manuscript submitted for publication.

Rice, D., \& Barone, S. J. (2000). Critical periods of vulnerability for the developing nervous system: Evidence from humans and animal models. Environmental Health Perspectives, 108, 511-533.

Rosenblatt, J. S., \& Lehrman, D. (1963). Maternal behavior of the laboratory rat. In H. L. Rheingold (Ed.), Maternal behavior in mammals (pp. 8-57). New York: Wiley.

Rosenblum, L. A., \& Paully, G. S. (1984). The effects of varying environmental demands on maternal and infant behavior. Child Development, $55,305-314$.

Ross, H. E., \& Young, L. J. (2009). Oxytocin and the neural mechanisms regulating social cognition and affiliative behavior. Frontiers in Neuroendocrinology, 30, 534-547.

Roth, T. L., \& Sweatt, J. D. (2012). Epigenetic mechanisms and environmental shaping of the brain during sensitive periods of development. Child Psychology and Psychiatry, 52, 398-408.

Sanchez-Andrade, G., \& Kendrick, K. M. (2009). The main olfactory system and social learning in mammals. Behavioural Brain Research, 200, 323-335.

Scheele, D., Wille, A., Kendrick, K. M., Stoffel-Wagner, B., Becker, B., \& Güntürkün, O. (2013). Oxytocin enhances brain reward system responses in men viewing the face of their female partner. Proceedings of the $\mathrm{Na}$ tional Academy of Sciences, 110, 20308-20313.

Schneiderman, I., Kanat-Maymon, Y., Ebstein, R. P., \& Feldman, R. (2013). Cumulative risk on the oxytocin receptor gene (OXTR) underpins empathic communication difficulties at the first stages of romantic love. Social Cognitive and Affective Neuroscience. Advance online publication.

Schneiderman, I., Kanat-Maymon, Y., Zagoory-Sharon, O., \& Feldman, R (2014). Mutual influences between partners' hormones shape conflict dialog and relationship duration at the initiation of romantic love. Social Neuroscience, 9, 337-351.

Schneiderman, I., Zagoory-Sharon, O., Leckman, J. F., \& Feldman, R. (2012). Oxytocin during the initial stages of romantic attachment: Relations to couples' interactive reciprocity. Psychoneuroendocrinology, 37, $1277-1285$.

Schneirla, T. (1946). Problems in the bio-psychology of social organization. Journal of Abnormal and Social Psychology, 41, 385.

Schneirla, T., \& Rosenblatt, J. S. (1963). "Critical periods" in the development of behavior. Science, 139, 110-115.

Scott, J. P. (1962). Critical periods in behavioral development critical periods determine the direction of social, intellectual, and emotional development. Science, 138, 949-958.

Shahrokh, D. K., Zhang, T.-Y., Diorio, J., Gratton, A., \& Meaney, M. J. (2010). Oxytocin-dopamine interactions mediate variations in maternal behavior in the rat. Endocrinology, 151, 2276-2286. doi:10.1210/en.2009-1271

Smith, A. S., \& Wang, Z. (2012). Salubrious effects of oxytocin on social stress-induced deficits. Hormones and Behavior, 61, 320-330.

Sokolowski, K., \& Corbin, J. G. (2012). Wired for behaviors: From development to function of innate limbic system circuitry. Frontiers in Molecular Neuroscience, 5,55 .

Spunt, R. P., Satpute, A. B., \& Lieberman, M. D. (2011). Identifying the what, why, and how of an observed action: An fMRI study of mentalizing and mechanizing during action observation. Journal of Cognitive Neuroscience, 23, 63-74.

Stern, D. (1977). The first relationship: Infant and mother. Cambridge, MA: Harvard University Press.
Stevens, H. E., Leckman, J. F., Coplan, J. D., \& Suomi, S. J. (2009). Risk and resilience: Early manipulation of macaque social experience and persistent behavioral and neurophysiological outcomes. Journal of the American Academy of Child \& Adolescent Psychiatry, 48, 114-127.

Sullivan, R. M., \& Holman, P. J. (2010). Transitions in sensitive period attachment learning in infancy: The role of corticosterone. Neuroscience \& Biobehavioral Reviews, 34, 835-844.

Szyf, M., McGowan, P., \& Meaney, M. J. (2008). The social environment and the epigenome. Environmental and Molecular Mutagenesis, 49, 46-60.

Takesian, A. E., \& Hensch, T. K. (2013). Balancing plasticity/stability across brain development. Progress in Brain Research, 207, 3-34.

Tarullo, A. R., \& Gunnar, M. R. (2006). Child maltreatment and the developing HPA axis. Hormones and Behavior, 50, 632-639.

Thelen, E., \& Smith, L. (1994). A dynamic systems approach to the development of cognition and action. Cambridge, MA: MIT Press.

Theodosis, D. T., Montagnese, C., Rodriguez, F., Vincent, J. D., \& Poulain, D. A. (1986). Oxytocin induces morphological plasticity in the adult hypothalamo-neurohypophysial system. Nature, 322, 738-740.

Thorpe, K. (2006). Twin children's language development. Early Human Development, 82, 387-395.

Tinbergen, N. (1963). On aims and methods of ethology. Animal Biology (Leiden), 55, 297-322.

Tottenham, N. (2014). The importance of early experiences for neuro-affective development. Current Topics in Behavioral Neurosciences, 16, 109-129.

Toyoizumi, T., Miyamoto, H., Yazaki-Sugiyama, Y., Atapour, N., Hensch, T. K., \& Miller, K. D. (2013). A theory of the transition to critical period plasticity: Inhibition selectively suppresses spontaneous activity. Neuron, 80, 51-63.

Uvnas-Moberg, K. (1997). Physiological and endocrine effects of social contact. Annals of the New York Academy of Sciences, 807, 146-163.

Weaver, I. C., Cervoni, N., Champagne, F. A., D'Alessio, A. C., Sharma, S. Seckl, J. R. et al. (2004). Epigenetic programming by maternal behavior. Nature Neuroscience, 7, 847-854.

Weisman, O., \& Feldman, R. (2013). Oxytocin administration affects the production of multiple hormones. Psychoneuroendocrinology, 38, 626-627.

Weisman, O., Granat, A., Gilboa-Schechtman, E., Singer, M., Gordon, I., Azulay, H. et al. (2010). The experience of labor, maternal perception of the infant, and the mother's postpartum mood in a low-risk community cohort. Archives of Women's Mental Health, 13, 505-513.

Weisman, O., Magori-Cohen, R., Louzoun, Y., Eidelman, A. I., \& Feldman, R. (2011). Sleep-wake transitions in premature neonates predict early development. Pediatrics, 128, 706-714.

Weisman, O., Schneiderman, I., Zagoory-Sharon, O., \& Feldman, R. (2013). Salivary vasopressin increases following intranasal oxytocin administration. Peptides, 40, 99-103.

Weisman, O., Zagoory-Sharon, O., \& Feldman, R. (2012). Oxytocin administration to parent enhances infant physiological and behavioral readiness for social engagement. Biological Psychiatry, 72, 982-989.

Weisman, O., Zagoory-Sharon, O., \& Feldman, R. (2013). Oxytocin administration alters HPA reactivity in the context of parent-infant interaction. European Neuropsychopharmacology, 23, 1724-1731.

Weisman, O., Zagoory-Sharon, O., \& Feldman, R. (2014). Oxytocin administration, salivary testosterone, and father-infant social behavior. Progress in Neuro-Psychopharmacology and Biological Psychiatry, 49, 47-52.

Wheeler, W. M. (1928). Emergent evolution and the development of societies. New York: Norton.

Wilson, E. O. (2012). The social conquest of earth. New York: Norton.

Winslow, J. T., Noble, P. L., Lyons, C. K., Sterk, S. M., \& Insel, T. R. (2003). Rearing effects on cerebrospinal fluid oxytocin concentration and social buffering in rhesus monkeys. Neuropsychopharmacology, $28,910-918$

Wynne-Edwards, K. E. (2001). Hormonal changes in mammalian fathers. Hormones and Behavior, 40, 139-145.

Yaniv, A., Avitsur, R., Kanat-Maymon, Y., Schneiderman, I., ZagoorySharon, O., \& Feldman, R. (2015). Affiliation, reward, and immune biomarkers increase and coalesce during periods of bond formation in humans. Manuscript submitted for publication.

Zheng, J.-J., Li, S.-J., Zhang, X.-D., Miao, W.-Y., Zhang, D., Yao, H. et al (2014). Oxytocin mediates early experience-dependent cross-modal plasticity in the sensory cortices. Nature Neuroscience, 17, 391-399. 